\title{
Adaptive Dynamics of Speciation: Sexual Populations
}

Ulf Dieckmann (dieckman@iiasa.ac.at)

Michael Doebeli (doebeli@zoology.ubc.ca)

\section{Approved by}

Leen Hordijk (hordijk@iiasa.ac.at)

Director, IIASA

November 2004 


\section{Contents}

1 Introduction 1

2 Adaptive Speciation in Sexual Populations 3

The obstructive role of segregation and recombination . . . . . . . . . . . 3

An overview of earlier studies . . . . . . . . . . . . . . . 5

Asexual adaptive speciation through resource competition . . . . . . . . . . . 9

No adaptive speciation under random mating . . . . . . . . . . . . . . . . . 10

Adaptive speciation through evolution of assortative mating . . . . . . . . . . 10

3 Coevolutionary Adaptive Speciation in Sexual Populations 15

Adaptive speciation through mutualistic interactions . . . . . . . . . . . . . . . 15

Adaptive speciation through predator-prey interactions . . . . . . . . . . . 16

4 Adaptive Speciation through Sexual Selection 18

Adaptive speciation through mate competition . . . . . . . . . . . . . . . . . . . . . 19

Adaptive speciation through sexual conflict . . . . . . . . . . . . . . 21

5 Concluding Comments 25 


\author{
About the Authors \\ Ulf Dieckmann \\ Adaptive Dynamics Network \\ International Institute for Applied Systems Analysis \\ A-2361 Laxenburg, Austria \\ Michael Doebeli \\ Department of Zoology \\ University of British Columbia \\ 6270 University Blvd. \\ Vancouver BC V6T 1Z4, Canada
}

\title{
Acknowledgments
}

Ulf Dieckmann gratefully acknowledges financial support from the Austrian Science Fund, from the Austrian Federal Ministry of Education, Science, and Cultural Affairs, and from the European Research Training Network ModLife (Modern Life-History Theory and its Application to the Management of Natural Resources), funded through the Human Potential Programme of the European Commission.

Michael Doebeli gratefully acknowledges financial support from the National Science and Engineering Council (NSERC) of Canada, and from the James S. McDonnell Foundation, USA. 


\title{
Adaptive Dynamics of Speciation: Sexual Populations
}

\author{
Ulf Dieckmann \\ Michael Doebeli
}

\section{Introduction}

When John Maynard Smith (1966) wrote on sympatric speciation more than 35 years ago, he acknowledged that the argument "whether speciation can occur in a sexually reproducing species without effective geographical isolation" was an old problem and voiced his opinion that the "present distribution of species is equally consistent either with the sympatric or the allopatric theory." Yet, from the heyday of the Modern Synthesis until relatively recently, the importance of sympatric speciation has been downplayed, and the corresponding hypotheses remained obscure well beyond Maynard Smith's seminal study.

Looking back from today's perspective, it is astounding that, for such a long period, the research community at large essentially turned a blind eye to sympatric speciation. Given the widely acknowledged difficulties involved in inferring past process from present pattern, one can only feel uneasy about a logic that claims to find evidence for the prevalence of allopatric speciation in the present-day distribution of species. To a large extent it seems to have been the scientific community's perception of the theory of sympatric speciation that has brought about a profound skepticism toward the broader empirical relevance of this speciation mode. Scientific attempts to overcome this skepticism have come and gone in waves. In the 1960s, luminaries of North American evolutionary biology pulled no punches when assessing the merit of such attempts. Displaying a characteristic hint of restrained intimidation, Ernst Mayr (1963) wrote on sympatric speciation, "One would think that it should no longer be necessary to devote much time to this topic, but past experience permits one to predict that the issue will be raised again at regular intervals. Sympatric speciation is like the Lernaean Hydra which grew two new heads whenever one of its old heads was cut off." And also Theodosius Dobzhansky's verdict was categorical when he remarked, in 1966, that "sympatric speciation is like the measles; everyone gets it and we all get over it" (Bush 1998). Sometimes models of sympatric speciation were interpreted to imply that such speciation was only possible under very special and narrow conditions, while at other times the same models were called into question as they allegedly predicted sympatric speciation to happen too easily. In the words of Felsenstein (1981), "one might come away from some of these papers with the disturbing impression that [sympatric speciation] is all but inevitable." Such concerns were echoed again recently, by Bridle and Jiggins (2000), for example.

Sympatric speciation is contingent on constraints that are both ecological and genetic. An appreciation of the complementary character of these constraints helps to explain how the orientation of researchers toward ecological or genetic detail influenced their views on sympatric speciation. For instance, while Maynard Smith (1966) emphasizes that the "crucial step in sympatric speciation is the establishment of a stable polymorphism", Felsenstein (1981) contends that progress toward sympatric speciation ought to be measured in terms of the evolution of prezygotic isolation. Felsenstein even goes so far as to argue that, without genetic constraints on speciation, we ought to expect "a different species on every bush", and thus implies that the corresponding ecological 
constraints are fulfilled trivially. It seems that this latent disciplinary divide has not helped the subject, and lingers on today.

Perhaps the majority view of the past decades can be summarized crudely by four brief statements. First, most biologists remained deeply skeptical about sympatric speciation, in recognition that its relevance was highly contentious. Second, they believed that the ecological constraints for speciation to occur in sympatry were still somewhat more restrictive than admitted by proponents of this mode. Third, they insisted that the true and central challenge was to explain the emergence of reproductive isolation in sexual species and, fourth, there was a widespread impression that models of sympatric speciation failed as soon as genetic constraints were accounted for adequately.

Recent theoretical research is contributing to overcoming these concerns. Chapter 4 in Dieckmann et al. 2004 describes why the phenomenon of evolutionary branching through frequencydependent selection provides a unifying framework within which to understand the ecological constraints on sympatric speciation - by explaining the dynamic emergence and subsequent perpetuity of disruptive selection in speciation events.

This chapter, in turn, shows how the genetic constraints on sympatric speciation are overcome more readily than earlier work had us believe. Thus, we claim no less than that previous reservations based on theoretical difficulties turn out to be unfounded upon closer, and more elaborate, inspection of the issues. We note that virtually all known theoretical examples, old and new, of sympatric speciation arise in the context of disruptive selection induced by frequency-dependent selection. Therefore, in adherence with our tenet that speciation research would benefit from concentrating on processes and mechanism, rather than on biogeographic patterns alone (Chapter 1 in Dieckmann et al. 2004), all the examples reviewed in this chapter must be recognized as representing instances of adaptive speciation. However, out of respect for the tradition of the field we retain, for the most part, the classic terminology of "sympatric" speciation. Section 2 starts out with a detailed review of the relevant genetic constraints and thus highlights the particular challenges that models of sympatric speciation have to meet if they are to be applied to sexual populations. After a short historical overview, we discuss ecologically and genetically explicit models that extend beyond the simplicity of earlier genetic studies. Two extensions are key:

- Earlier models involved only a few loci (usually two or three), each of which coded for a different phenotypic component through a very small number of alleles (usually just two). These simple approaches occasionally allowed some analytical treatment (e.g., reviewed in Christiansen and Feldman 1975), and were geared usefully to the capacities available for numerical simulation 20 years ago. Today, however, multilocus models that involve quasicontinuous quantitative characters seem more suitable and realistic.

- The deterministic nature of earlier models also turned out to be problematic. Today, individual-based stochastic approaches are becoming increasingly tractable numerically. These latter models can exhibit dynamics qualitatively different from those predicted by their deterministic counterparts (Dieckmann and Doebeli 1999). This is not surprising since deterministic models, strictly speaking, only correctly describe infinitely large populations that do not contain ecological and genetic drift.

Section 2 concludes with a demonstration of how avoidance of these two pitfalls of oversimplification changes our views about the restrictiveness of genetic constraints on sympatric speciation. Underscoring the same general message again from the ecological end, Section 3 illustrates how easily a wide variety of ecological settings, which involve all three fundamental types of ecological interaction, can induce evolutionary branching in sexual populations.

Evolution in sexual populations is not always more restricted than in asexual ones. For models of sympatric speciation, the additional options for the emergence of prezygotic isolation presented 
by adaptation in sexual traits are especially interesting. In particular, it has been suggested that divergent Fisherian runaway processes in sexual traits can cause sympatric speciation, even in the absence of disruptive selection on any ecological character. Section 4 features a summary of these recent developments and explains how sexual selection and sexual conflict can facilitate speciation in sympatry. Also in such processes, frequency-dependent disruptive selection plays a central role, which reveals a fundamental similarity of the underlying mechanisms. Sympatric speciation driven by such frequency-dependent selection on ecological and sexual traits is adaptive (Chapters 1 and 19 in Dieckmann et al. 2004), and allows either an entire population or the separate sexes to escape from fitness minima.

\section{Adaptive Speciation in Sexual Populations}

The evolutionary force that favors sympatric speciation is disruptive selection, while the forces that oppose it, generally speaking, are segregation and recombination (Felsenstein 1981). The ecological constraints on sympatric speciation are therefore essentially given by the conditions required for a population to be exposed to disruptive selection for a sufficiently long period (Chapter 4 in Dieckmann et al. 2004), whereas the genetic constraints originate from a need to overcome segregation and recombination before a sexual population can split sympatrically (and potentially undergo character displacement). After examining in some detail the specific challenges posed to sympatric speciation by segregation and recombination, and after providing an overview of earlier work on this topic, this section describes the corresponding remedies.

\section{The obstructive role of segregation and recombination}

Speciation in sympatry requires reproductive isolation to arise between two incipient species, and so overcome the cohesion of a species' gene pool caused by segregation and recombination. In the initial phases of sympatric speciation, the divergent subpopulations are often separated by postzygotic isolation in the form of hybrid inferiority through disruptive selection. Enhancement of such initial, ecologically inflicted postzygotic isolation would typically occur through prezygotic isolation (see Box 6.1 in Dieckmann et al. 2004). Hence, the evolution of prezygotic isolation, through assortative mating in the broadest sense, lies at the core of the problem of segregation and recombination obstructing sympatric speciation in sexual populations. Assortativeness can either be tied directly to the species' ecology that causes the disruptive selection, or evolve independently. A well-known example of the former case is the famous Rhagoletis system (Bush 1975; Feder et al. 1988), in which the preferences of maggot flies for apple or hawthorn trees not only determine their feeding grounds, but also simultaneously restrict the types of mate they are likely to encounter. A typical example for the latter case is an ecologically neutral mating preference based on courtship behavior.

Below we consider general models characterized by two sets of loci that code, respectively, for an ecologically relevant phenotype (E-loci) and for assortative mating behavior (A-loci). Felsenstein (1981) focused on models that involved one E-locus and one A-locus. On this basis he introduced an important distinction between "speciation in which the reproductive isolating mechanisms come into existence by the substitution of different alleles in the two nascent species, and speciation in which the same alleles are substituted in both species." Felsenstein (1981) refers to these cases as "two-allele" and "one-allele" models, respectively. In "one-allele" models of sympatric speciation that involve two patches, the A-locus could code, for example, for the degree of patch philopatry or for the probability with which individuals settle in the patch they are best adapted to. By either means, two incipient species might evolutionarily restrict their mixing between the two patches, and thus enhance prezygotic isolation by exhibiting the same allele at 
their A-locus. In both cases, the degree of choice is determined by the A-locus, while the choice itself is pleiotropically affected by the E-locus. By contrast, in "two-allele" models the A-locus codes for the target of mate choice itself, such as for the chosen mating patch or for the partner's ecological phenotype, which requires incipient species to carry different alleles at this locus.

Despite its importance, the allele-centric terminology Felsenstein (1981) chose for his simple models is not ideal for a variety of reasons:

- When considering sympatric speciation in multilocus models, allele substitutions may be required at many loci, and thus necessarily involve more than just one or two alleles.

- The term "two-allele" model is potentially quite confusing in the context of models with diallelic loci.

- It is actually immaterial whether alleles involved in substitutions are genotypically identical or different - it is only their effects on the mating phenotype that matter.

For these reasons it would be more accurate and less confusing to refer to Felsenstein's "oneallele" models as requiring concordant allele substitutions on the A-loci, while his "two-allele" models can lead to sympatric speciation only through discordant substitutions on these loci. Yet, since Felsenstein's terminology has become well established in the thinking of speciation scientists, we continue to refer to "one-allele" and "two-allele" models below, but the three provisos and the extended multilocus meaning of these traditional terms must be kept firmly in mind.

Returning to the general case that involves arbitrary numbers of E- and A-loci, we can now unravel the role of segregation and recombination in sympatric speciation processes into three components:

- Segregation and recombination among E-loci. Intermediate ecological phenotypes can arise whenever two or more ecological alleles are involved per individual: they are generated through recombination if there is more than one E-locus, and through segregation if inheritance is diploid. For most genotype-to-phenotype maps a depression in the frequency of intermediates is swamped rapidly by offspring from parents with ecological phenotypes from either mode, which thus causes the modes to coalesce. Too much segregation or recombination, therefore, is bound to prevent the evolution of any bimodality in the ecological character. At the same time, however, some intermediates are required for the population to continue to experience the consequences of hybrid inferiority, which potentially increases prezygotic isolation through evolution on the A-loci. Therefore, a certain degree of segregation and/or recombination is indispensable for the evolution of prezygotic reproductive isolation during processes of sympatric speciation.

- Segregation and recombination between E- and A-loci. In "two-allele" models - in which the E-loci affects the degree of postzygotic isolation and the A-loci that of prezygotic isolation - recombination and segregation tend "to break down the association between the prezygotic and postzygotic isolating mechanisms, so that it is always eroding the degree of progress toward speciation" (Felsenstein 1981). Again, therefore, segregation and recombination obstruct the speciation process. Sometimes, however, segregation and/or recombination between E- and A-loci are even needed for sympatric speciation. This is the case in a model by Dieckmann and Doebeli (1999) in which E-loci code for an ecological character under disruptive selection and A-loci determine an ecologically neutral marker character on which mate choice is based. In a process of selection-enhanced symmetry breaking (as explained below in the context of Figure 4a), segregation and recombination cause the correlation between ecological and marker characters to fluctuate enough for selection to maximize that linkage disequilibrium.

- Segregation and recombination among A-loci. Also for the loci involved in assortative mating, recombination and segregation can lead to the formation of intermediates. In "one- 
allele" models, recombination or segregation between the A-loci is not an issue since the corresponding alleles are expected to undergo concordant substitutions throughout the speciation process. The situation is very different in "two-allele" models, since here recombination among A-loci can create intermediates that suffer from decreased mating frequencies with partners from both incipient species, which thus introduces a cost to assortativeness that may prevent its evolutionary emergence.

This decomposition of effects allows us to draw the following conclusions:

- It is only in trivial haploid models with a single E-locus and a "one-allele" mechanism at the A-loci that segregation and recombination do not impede sympatric speciation.

- In all other "one-allele" models, segregation and recombination selectively favor the evolution of assortative mating because of hybrid inferiority, but hinder the evolution of bimodality in the ecological character. This means, incidentally, that the assertion by Felsenstein (1981) that only in "two-allele" models "does recombination act as a force to retard or block speciation" does not carry over to multilocus models.

- In all "two-allele" models, sympatric speciation is further obstructed by segregation and recombination between E- and A-loci, as well as among A-loci. At the same time, however, in some of these models the emergence of linkage disequilibria through selection-enhanced symmetry breaking relies on the fluctuations caused by segregation and recombination in finite populations.

Notice that the effects of segregation and recombination become more subtle if there is epistasis between the E- and A-loci, or if the E- or A-loci code for more than one quantitative character each. In the latter case, sympatric speciation may require some of these quantitative characters to evolve through concordant allele substitutions, while for other characters substitutions ought to be discordant. In such general cases, segregation and recombination have two effects: first, they potentially obstruct bimodalities in the individual characters that ought to undergo discordant substitutions, and second, they may weaken linkage disequilibria in pairs of such characters.

\section{An overview of earlier studies}

Many key ideas about sympatric speciation can be traced back to landmark papers by Maynard Smith (1966), Rosenzweig (1978), and Felsenstein (1981). Especially when the effects of segregation and recombination, described above, are considered these and other analyses offered divergent perspectives on the relative importance and implications of ecological and genetic constraints on the feasibility of sympatric speciation.

The pioneering study by Maynard Smith (1966) emphasized the ecological conditions required for the evolution, through hybrid inferiority, of stable polymorphisms between incipient species in two-niche models of soft selection (Levene 1953; see also Chapter 3 in Dieckmann et al. 2004 and Kisdi 2001). Based on diploid genetics with one E-locus and at most one A-locus, it was concluded that "the conditions which must be satisfied are [...] severe": density regulation must operate separately in the two niches, and the advantages of local adaptation to either niche must be large. Maynard Smith (1996) also put forward four mechanisms for the evolution of reproductive isolation:

- Habitat choice - implying assortative mating as a by-product of an individual's fidelity to or preference for the habitat experienced after birth, and envisaged in terms of a "one-allele" model.

- Pleiotropism - in which an allele that affects the ecological character under disruptive selection itself causes assortative mating, an option that does not require any A-loci and that Maynard Smith considered unlikely to occur in nature. 
- Modifier genes - a "one-allele" mechanism of assortative mating on the ecological character, in which the responsible allele is assumed to be dominant.

- Assortative mating genes - a "two-allele" mechanism of assortative mating in which one of the two involved alleles is again assumed to be dominant.

Assessing the resultant genetic constraints for sympatric speciation, Maynard Smith admitted that for the "one-allele" and "two-allele" mechanisms to work, they must be supported by habitat choice. Also, without analyzing the "two-allele" model in full, he concluded that in such models habitat choice and direct assortative mating must be accurate enough and disruptive selection must be sufficiently strong for sympatric speciation to be initiated.

Maynard Smith's focus on Levene-type models of soft selection has been retained in most subsequent studies of sympatric speciation, as reviewed in Chapter 3 in Dieckmann et al. 2004. In particular, the synergistic interactions between habitat choice and "two-allele" mechanisms of assortative mating observed by Maynard Smith are highlighted in Section 3.5 in Dieckmann et al. 2004; see also Kawecki $(1996,1997)$ and Johnson et al. (1996b). Note that Maynard Smith himself had not yet presented a perspective in which the issue of segregation or recombination between Eand A-loci (especially in "two-allele" models) was placed at center stage.

"Two-allele" mechanisms of assortative mating also did not figure in an analysis by Dickinson and Antonovics (1973), which probed Maynard Smith's extension of Levene's model in greater detail. Instead, these authors explored, again based on simple diploid two-niche models of soft selection, the evolution of polygenic ecological characters coded for by up to three diallelic Eloci, of linkage between the involved loci, and of dominance relations between the involved alleles. They also considered an extra diallelic A-locus that determined selfing or assortative mating, but only according to "one-allele" mechanisms. Dickinson and Antonovics (1973) showed that a polymorphism could be maintained with strong selection and/or low levels of gene flow between the niches, and that modifiers for linkage, dominance, selfing, and assortativeness could spread.

Speciation models based on multiple discrete niches are exposed occasionally to the criticism that what they really model is not sympatric, but at best microallopatric, or - if the niches considered are not finely entangled spatially - even parapatric speciation. A central contribution to the debate about the wider relevance of sympatric speciation was therefore Rosenzweig's (1978) model of competitive speciation, in which he argued that - even in a continuously structured niche space and when considering the evolution of a quantitative character in such a space - intraspecific resource competition could be expected to induce speciation. Rosenzweig (1978) stressed the fundamental capacity of frequency-dependent selection to re-mold the shape of fitness landscapes (e.g., as illustrated in Figures 1.1 in Dieckmann et al. 2004 and 1a): when an evolving population has attained a location on a fitness landscape that would be a peak in the absence of intraspecific competition, such competition can impose a dimple on the fitness landscape at the crowded location, as a result of which selection there becomes disruptive. This mechanism considerably broadens the range of ecological settings that could be expected to facilitate sympatric speciation. Taking an ecological perspective, Rosenzweig (1978) remained characteristically unconcerned about the evolution of reproductive isolation: mechanisms of assortative mating are not even mentioned, and effects of habitat choice are only discussed in passing.

A change in tack occurred with the studies of Udovic (1980), Felsenstein (1981), and Seger (1985a). All three articles focused on the conditions required for the evolution of prezygotic reproductive isolation driven by disruptive selection, and all explored "two-allele" mechanisms of assortative mating in a potentially panmictic population. The investigations show further similarities as they all relied on simple genetic models that involve one or two diallelic E- and A-loci, and thus on a coarse array of phenotypes. Another parallel between the setup of these models is that no provision was made to let the degree of assortative mating evolve gradually: usually, one allele was assumed to code for random mating behavior, while the other caused assortativeness 
(a)

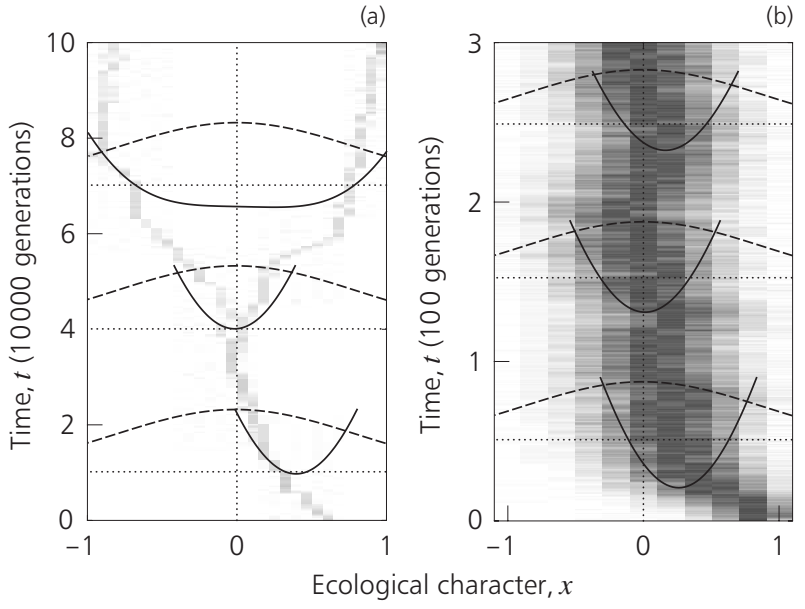

Figure 1 Evolutionary convergence to a fitness minimum. Gray scales indicate the frequency of phenotypes (the darkest shade corresponds to the highest frequency). (a) The asexual population first evolves toward $x_{0}=0$, the maximum of the carrying capacity $K(x)$ (dashed curves). Once this maximum is reached, directional selection turns disruptive, and the population finds itself exposed to a fitness minimum. As a result, the previously unimodal population becomes bimodal, and splits into two morphs through evolutionary branching. The overlays show the invasion fitness of mutant phenotypes (continuous curves) as generated by the ecological interactions with the resident phenotypes at three different points in time (indicated by horizontal dotted lines). (b) As in (a), but with multilocus genetics for the ecological character and with random mating. Shades of gray represent the phenotype distributions: with $l_{\mathrm{E}}=5$ diploid and diallelic loci, 11 ecological phenotypes can arise. Despite the continual action of disruptive selection at $x_{0}=0$, as shown by the invasion fitness of asexual mutants (continuous curves), segregation and recombination among the E-loci prevent the occurrence of evolutionary branching. For this reason, the sexual population remains trapped at the fitness minimum. Other parameters: $r=1, K_{0}=500, \sigma_{K}=2, \sigma_{a}=0.8, \sigma_{\mathrm{as}}=210^{-2}$, $u_{\mathrm{as}}=u_{\mathrm{s}}=10^{-3}$. Source: Dieckmann and Doebeli (1999).

at an externally fixed level. While Seger (1985a) focused on haploid inheritance, Udovic (1980) analyzed diploid genetics. Felsenstein (1981) mostly concerned himself with modeling haploids, but also presented results for the diploid case.

For one E-locus and one A-locus, Udovic (1980) investigated in detail how, in a diploid model, the reproductive isolation between two incipient species depended on the degrees of imposed disruptive selection, of frequency-dependent selection, of assortative mating, and of recombination. He concluded that the evolution of prezygotic isolation is constrained by a lower bound on the intensity of disruptive selection, and that the threshold value increased with the assumed recombination fraction and decreased with the amount of assortativeness assumed to be conferred by one of the alleles.

The seminal analysis by Felsenstein (1981) set the agenda for discussing sympatric speciation models for the subsequent two decades. Felsenstein numerically analyzed a haploid model with two E-loci and one A-locus by determining the degrees of disruptive selection and of assortative mating that would allow prezygotic isolation to become established. His results are in agreement with those of Udovic (1980), especially with regard to a "complex trade-off" between the strength of disruptive selection and the accurateness of assortative mating required for speciation. Felsenstein (1981) also looked at the robustness of his results with regard to recombination fractions, migration rates between the two niches, reversing the sequence of mating and migration in the species' life cycle, epistasis between the ecological loci, diploidy, and evolution at a modifier locus, which resulted in two diallelic A-loci and allowed three different levels of assortative mating. He concluded that "selection is at risk of being overwhelmed by recombination" and that "selection can proceed only when there is sufficiently strong selection at [the ecological loci], or sufficiently weak gene flow between the two nascent species." 
Seger (1985a) studied a haploid model that also featured two diallelic E-loci and one A-locus. Perhaps inspired by Rosenzweig (1978), and in contrast to the analyses by Udovic (1980) and by Felsenstein (1981), Seger considered a more explicit and mechanistic ecological underpinning, based on intraspecific resource competition and involving ecological phenotypes derived from the corresponding genotypes by the imposition of environmental variation. Seger (1985a) assumed that this variation broadened the range of three ecological genotypes to five ecological phenotypes, which corresponded to adjacent intervals along a continuous resource axis. He also assumed that resource competition would only operate within these intervals, and considered different resource distributions across the intervals. He showed that the resultant frequency-dependent selection pressures could lead to sympatric speciation if the distribution of resources was overdispersed slightly relative to the phenotypic distribution that would occur under panmixia. Under this condition, "one-allele" and "two-allele" mechanisms of assortative mating could evolve. Seger (1985a) also investigated an extended scheme with two A-loci, in which one diallelic A-locus coded for an ecologically neutral marker trait while the other A-locus determined whether or not mating was assortative on that character. Although sympatric speciation could occur under the latter scheme (albeit very slowly), Seger expressed reservations about the conditions required and concluded that prezygotic isolation under such circumstances could not be expected to arise spontaneously.

It seems likely that Seger's results were affected by the artificial discretization of the resource space and by the low resolution of his set of genotypes. Two remedies are available for this problem: either a multitude of alleles can be considered, or many loci can be assumed to affect the characters under selection. When Kisdi and Geritz (1999) and Geritz and Kisdi (2000) re-analyzed the evolution of specialization in Levene's model of soft selection, they assumed a single E-locus, but (unlike previous work) they considered an infinite range of potential alleles on that locus. Envisaging that the evolution of these alleles would proceed by rare mutations that cause small steps in the ecological character only, Kisdi and Geritz (1999) showed the ecological constraints on sympatric speciation to be much less restrictive than previous research based on diallelic loci had suggested (see Box 4.7 in Dieckmann et al. 2004).

Kondrashov (1983a, 1983b) was the first to introduce diploid multilocus genetics systematically into models of sympatric speciation (see also Kondrashov 1986; Kondrashov and Mina 1986). Kondrashov's models do not use an explicit ecological embedding to provide a mechanistic and dynamic basis for regimes of disruptive selection: instead, an unspecified cause is assumed to favor marginal phenotypes at all times. Kondrashov's early studies considered only E-loci: the type and degree of assortative mating was not modeled as a quantitative character, and thus the evolution of assortativeness was not actually investigated. Kondrashov and Shpak (1998) showed that the types of assortative mating that can cause sympatric speciation when the ecological character is turned into an ecologically neutral trait are rather limited. Kondrashov and Kondrashov (1999) incorporated A-loci into the earlier models. In one scenario, these extra loci code for two quantitative characters that describe an ecologically neutral male trait (A1-loci) and a female preference for a male trait value (A2-loci). In a simplified scenario, only the male trait was modeled, while the male trait preferred by a female was supposed to be the trait it would display as a male. Sympatric speciation was found to occur in both scenarios, facilitated by strong disruptive selection, a high number of E-loci, and a low number of A-loci. Notice that in none of these models was the choosiness of female preference allowed to evolve - instead, a preexisting mechanism of assortative mating was assumed to operate before, throughout, and after the speciation process.

Work by Dieckmann and Doebeli (1999) integrated previous advances in the theory of sympatric speciation into a single framework. To investigate conditions for sympatric speciation in sexual populations, Dieckmann and Doebeli (1999) used a generic ecological embedding with frequency-dependent selection that arises from intraspecific resource competition (like Rosenzweig and Seger), considered mechanisms of assortative mating that required either concordant 
or discordant allele substitutions (like Felsenstein and Udovic), employed explicit multilocus genetics (like Kondrashov) for separate ecological and mating characters, and incorporated these elements into individual-based stochastic population dynamics. These models and the resultant findings are summarized in the following three subsections.

\section{Asexual adaptive speciation through resource competition}

We start from ecological assumptions that are likely to be satisfied in many natural populations. Individuals vary in an ecological character $x$ that characterizes their resource utilization, such as when beak size in birds determines the size of seeds they can best consume. Populations that consist of individuals of a given trait value $x$ have density-dependent logistic growth with carrying capacity $K(x), \mathrm{d} n / \mathrm{d} t=r n[1-n / K(x)]$, where $n$ is the population's density. Individuals give birth at a constant rate $r$ and die at a rate determined by frequency- and density-dependent competition. The resource distribution and thus the carrying capacity $K(x)$ are assumed to be unimodal (a multimodal resource distribution can be partitioned into unimodal segments, which may be analyzed separately). The carrying capacity depends on the ecological character $x$ and varies according to a Gaussian function, $K(x)=K_{0} \exp \left(-\frac{1}{2}\left(x-x_{0}\right)^{2} / \sigma_{K}^{2}\right)$, which peaks at an intermediate phenotype $x_{0}$ and has variance $\sigma_{K}^{2}$. The stable equilibrium density of a population monomorphic in $x$ is thus $\hat{n}=K(x)$. Without loss of generality, we chose $x_{0}=0$.

In polymorphic populations that consist of subpopulations with different trait values $x_{i}$ and population densities $n_{i}$, dissimilar individuals interact only weakly, as, for example, when birds with different beak sizes eat different types of seeds. This implies that competition is not only density-dependent, but also frequency-dependent, and rare phenotypes experience less competition than common phenotypes. Specifically, we assume that the strength of competition between individuals declines with phenotypic distance $\Delta x$ according to a Gaussian function $a(\Delta x)=\exp \left(-\frac{1}{2} \Delta x^{2} / \sigma_{a}^{2}\right)$ that peaks at 0 and has variance $\sigma_{a}^{2}$. Polymorphic population dynamics are then described by

$$
\frac{\mathrm{d} n_{i}}{\mathrm{~d} t}=r n_{i}\left[1-\tilde{n}_{i} / K\left(x_{i}\right)\right],
$$

where the effective population density that affects individuals with ecological character $x_{i}$ is given by $\tilde{n}_{i}=\sum_{j} a\left(x_{j}-x_{i}\right) n_{j}$, that is, by summing over all other competitors while weighting their impact in accordance with the competition function $a$ (see Box 4.1 in Dieckmann et al. 2004 for an analysis of this model, as well as for references to the extensive earlier literature).

As a first step in our investigation, these assumptions are integrated into an asexual individualbased model, in which each individual is characterized by its trait value $x$ (for implementation details, see Box 7.1 in Dieckmann et al. 2004). Evolutionary dynamics occur because offspring phenotypes may deviate slightly from parent phenotypes (offspring have the same ecological character as their parent, except when a mutation occurs at rate $u_{\text {as }}$, in which case their character is chosen from a normal distribution that peaks at the parent's character and has variance $\sigma_{\text {as }}^{2}$ ). The quantitative character first evolves to the value $x_{0}=0$, which confers maximal carrying capacity. After that, two things can happen: either $x_{0}$ is evolutionarily stable and evolution halts at this point, or $x_{0}$ is actually a fitness minimum and can be invaded by all nearby phenotypes. In the latter case, evolutionary branching occurs as shown in Figure 1a. This happens for $\sigma_{a}<\sigma_{K}$, that is, if the curvature of the carrying capacity at its maximum is less than that of the competition function. Under this condition the advantage of deviating from the crowded optimal phenotype $x_{0}$ more than compensates for the disadvantage of a lower carrying capacity.

The incidence of evolutionary branching observed in the individual-based asexual model can be predicted as follows. When a rare mutant $x^{\prime}$ appears in a population that is monomorphic for the ecological character $x$ at carrying capacity $K(x)$, it competes with the discounted density 
$a\left(x-x^{\prime}\right) K(x)$, and the per capita growth rate $f\left(x^{\prime}, x\right)$ of the rare mutant $x^{\prime}$ (i.e., its invasion fitness, see Section 4.2 in Dieckmann et al. 2004) is given by $r\left[1-a\left(x-x^{\prime}\right) K(x) / K\left(x^{\prime}\right)\right]$ (see also Box 4.1 in Dieckmann et al. 2004). The derivative $g(x)$ of $f\left(x^{\prime}, x\right)$ with respect to the mutant character $x^{\prime}$, evaluated at the resident character $x, g(x)=\partial f\left(x^{\prime}, x\right) /\left.\partial x^{\prime}\right|_{x^{\prime}=x}=r K^{\prime}(x) / K(x)$, is positive for $x<x_{0}$ and negative for $x>x_{0}$. Therefore, $x_{0}$ is an attractor for the monomorphic adaptive dynamics. In addition, if $f\left(x^{\prime}, x\right)$ has a minimum at $x^{\prime}=x_{0}$, then $x_{0}$ is an evolutionary branching point (Box 4.3 in Dieckmann et al. 2004). The fitness minimum occurs if and only if $\sigma_{a}<\sigma_{K}$.

As mentioned above, a verbal account of this scenario of sympatric speciation in an asexual population had already been provided by Rosenzweig (1978), who remarked on the ecological character $x_{0}$ focusing utilization on the carrying capacity's peak: "[I]f its degree of specialization is sufficient relative to the breadth of its original Wrightian peak, it has created a dimple in the surface: the fitness of phenotypes to its left and right are higher than its own." The "surface" here has become formalized as the invasion fitness $f$ in adaptive dynamics theory (see Chapter 4 in Dieckmann et al. 2004). The "breadth of its original Wrightian peak" has to be equated with the width $\sigma_{K}$ of the carrying capacity function $K$, which remains unaffected by density regulation. And finally, the character's "degree of specialization" is measured by the width $\sigma_{a}$ of the competition function $a$. Although Rosenzweig's pioneering work did not anticipate the abstraction and generality associated with the phenomenon of evolutionary branching, which can originate from many causes other than resource competition, he had already captured the conceptual essence of evolution in the resource utilization model described above.

\section{No adaptive speciation under random mating}

As a second step, sexual reproduction is incorporated into the model by assuming that the ecological character $-1 \leq x \leq+1$ is determined by $l_{\mathrm{E}}$ equivalent diploid and diallelic E-loci with additive effects and free recombination. With a small probability $u_{\mathrm{s}}$, a mutation occurs in the inherited alleles and reverses their value. Only female individuals are modeled, while the genotypic distribution of males across the considered loci is assumed to match that of females. Alternatively, one could look upon this model as describing hermaphrodites.

In both the sexual and the asexual case, the population evolutionarily shifts its mean phenotype to $x_{0}$. However, if mating in the sexual population is random, evolutionary branching does not occur for any values of $\sigma_{K}$ and $\sigma_{a}$ (Figure 1b). As explained above, the evolution of two modes in the frequency distribution of the ecological character is prevented by the continual generation of intermediate phenotypes through segregation and recombination - even though, as shown in Figure $1 \mathrm{~b}$, the population continues to experience the disruptive selection associated with its exposure to a fitness minimum.

\section{Adaptive speciation through evolution of assortative mating}

To model the evolution of assortative mating, and thus of prezygotic isolation, we assume, as a third step, that individuals express additional quantitative characters that determine mating probabilities according to two scenarios:

- In the first scenario, mating probabilities are based on similarity in the ecological character $x$. The degree of choosiness involved in assortative mating is described by a mating character $y$ that is modeled as a second quantitative trait and is free to evolve.

- In the second scenario, mating probabilities are based on similarity in a third independent quantitative character $\tilde{x}$ that describes an ecologically neutral marker trait. In this latter scenario, both the level of choosiness in the mate choice and the marker trait itself can evolve. 


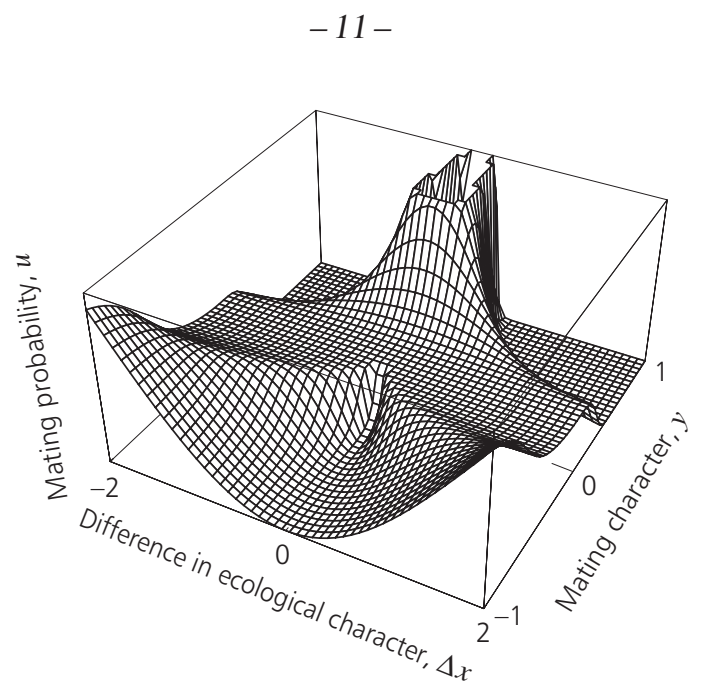

Figure 2 Modeling the degree of assortative mating. The mating character $-1 \leq y \leq+1$ is given by the difference between the number of + and - alleles divided by the total number of alleles at the A1-loci. For $y>0$, mating probabilities in the first scenario depend on the difference $\Delta x$ in the ecological trait according to $\exp \left(-\frac{1}{2} \Delta x^{2} / \sigma_{+}^{2}\right)$, with $\sigma_{+}=1 /\left(20 y^{2}\right)$. Hence, individuals that carry mostly + alleles at their A1-loci mate assortatively: the mating probability increases with phenotypic similarity to the partner. For $y=0$, mating is random (i.e., independent of the partner's ecological phenotype). For $y<0$, mating probabilities increase with the difference $\Delta x$ according to $1-\exp \left(-\frac{1}{2} \Delta x^{2} / \sigma_{-}^{2}\right)$, with $\sigma_{-}=y^{2}$. Individuals that carry mostly - alleles at their A1-loci thus mate disassortatively, being more likely to mate with partners with ecological phenotypes different from their own. In the second scenario, mating probabilities depend on the difference $\Delta \tilde{x}$ in an ecologically neutral marker trait instead of on $\Delta x$. To avoid a bias against marginal phenotypes, mating probabilities are normalized, so that their sum over all potential partners equals 1 for all phenotypes. Source: Dieckmann and Doebeli (1999).

Mating character $y$ and marker trait $\tilde{x}$ are underpinned by the same kind of multilocus genetics as the ecological character described before and are determined by $l_{\mathrm{A} 1}$ A1-loci and $l_{\mathrm{A} 2}$ A2-loci, respectively. The effect of the mating character is detailed in Figure 2; it allows the mating behavior of individuals to change gradually from negative assortativeness over random mating to positive assortativeness. Considering a mating character that describes the degree of choosiness enables us to study sympatric speciation without having to assume a preexisting level of assortative mating: initially our population is genetically coded to mate completely randomly.

Sympatric speciation in the first scenario requires concordant substitutions at the A1-loci toward higher degrees of choosiness, similar to Felsenstein's (1981) "one-allele" models. By contrast, the second scenario also necessitates concordant substitutions at the A1-loci, but in addition requires discordant substitutions at the A2-loci, at which a bimodal distribution of marker traits has to evolve and become correlated with the ecological character. The need for the incipient species to undergo such discordant allele substitutions means the second scenario is more akin to Felsenstein's (1981) "two-allele" models.

For the first scenario, Figure 3a shows the evolutionary dynamics of an initially randomly mating population that starts away from the evolutionary branching point. While the ecological character evolves toward $x_{0}$, the mating character initially changes only slowly, but picks up speed and evolves toward positive assortativeness when the mean of the ecological character reaches $x_{0}$. Once assortativeness is strong enough, the population splits into two ecologically different morphs, which eventually become almost completely reproductively isolated. As explained above, near the dynamically emerging fitness minimum at $x_{0}$, selection favors mechanisms that allow for a split in the phenotype distribution and hence for an escape from the fitness minimum: assortative mating is such a mechanism, because it prevents the generation of intermediate offspring phenotypes from extreme parent phenotypes. Figure 3 a shows that alleles which induce a high degree of positive assortative mating concordantly evolve in both incipient species. 
(a)

(b)


Figure 3 Adaptive speciation through resource competition, with assortative mating acting on the ecological character under disruptive selection. (a) The mean ecological character first evolves to the evolutionary branching point (50 generations); then the mean mating character increases to positive values (180 generations), which allows frequency-dependent disruptive selection to cause speciation (300 generations). Gray scales indicate the frequency of phenotypic combinations (black corresponds to the highest frequency). Source: Dieckmann and Doebeli (1999). (b) Combinations of standard deviations of carrying capacity and competition function that allow for adaptive speciation. Gray scales indicate the time to speciation (black corresponds to 0 generations and white to 20000 or more generations). In the corresponding asexual model, evolutionary branching happens for combinations that lie below the diagonal. Parameters are as in Figure 1, with $l_{\mathrm{A} 1}=5$. The asterisk in (b) shows the parameter combination used in (a).

Parameter requirements for evolutionary branching in the first scenario for the evolution of sexual populations are not much more demanding than those in the asexual case, as is shown in Figure $3 b$. There is one qualification, however. Selection for a polymorphic sexual population is disruptive only when the convolution $n * a$ of the phenotypic distribution $n(x)$ and the competition function $a(\Delta x)$ are narrower than the carrying capacity function $K(x)$ (G. Meszéna, personal communication). With Gaussian functions, this translates into the condition $\sigma_{a}^{2}+\sigma_{n}^{2}<\sigma_{K}^{2}$, where $\sigma_{n}^{2}$ is the variance of the phenotypic distribution when its mode has converged to $x_{0}$. Therefore, when $\sigma_{a}$ is just slightly smaller than $\sigma_{K}$, (i.e., when disruptive selection in the asexual model is weak), the sexual population may not actually experience disruptive selection at all (Matessi et al. 2001). Yet, as we can see in Figure 3b, in the setting we study here this effect does not lead to much of a reduction in the conditions that result in adaptive speciation in the first scenario.

Although Felsenstein's (1981) criticism of the biological relevance of "one-allele" models seems exaggerated - in particular because host races seem so widespread among insects (e.g., Berlocher and Feder 2002), and because assortativeness on body size is ubiquitous in animals (e.g., Schliewen et al. 2001) - it is nevertheless interesting to check whether the results described above carry over to the "two-allele" model in the second scenario. Felsenstein's (1981) general conclusion that sympatric speciation requiring discordant allele substitutions is very difficult was based crucially on his analysis of simple deterministic models that involved only two loci and two alleles per locus. We thus have to ask whether Felsenstein's (1981) time-honored conclusion stands up in the less restrictive context of quasi-continuous characters and stochastic multilocus genetics. As is shown below, the answer is negative.

For the second scenario, Figure 4a illustrates that in this case also the selective amplification of ecological and genetic drift in finite populations readily leads to speciation, despite the opposing forces of segregation and recombination. Such drift temporarily results in small and localized linkage disequilibria between some A2-loci and some E-loci. Both positive and negative correlations select for assortative mating, which in turn magnifies the local disequilibria into a global linkage disequilibrium between ecological character and marker trait. This feedback eventually induces a sympatric split into reproductively isolated phenotypic clusters. Thus, stochastic fluctuations in finite populations can spontaneously break the symmetry of locally stable linkage equilibria 

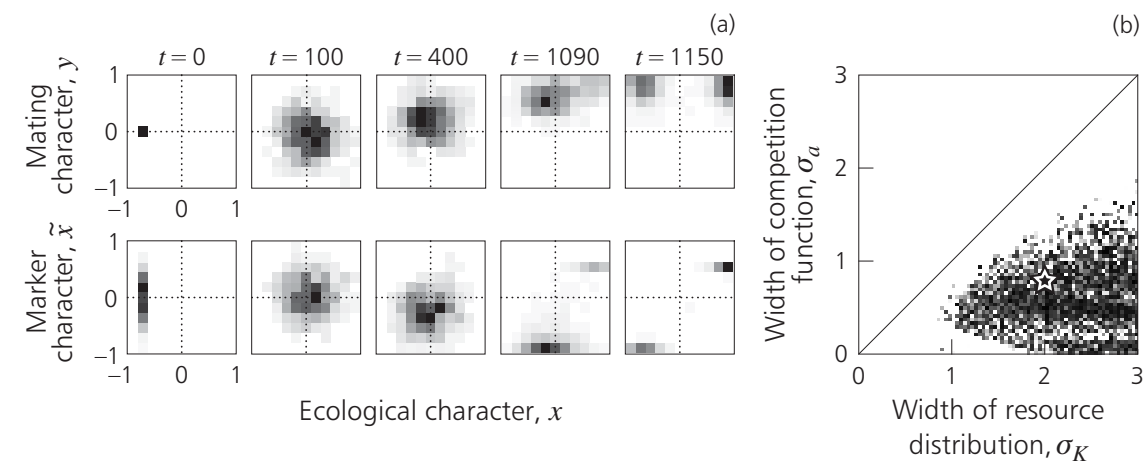

Figure 4 Adaptive speciation through resource competition, with assortative mating acting on an ecologically neutral marker trait. (a) The mean ecological character first evolves to the evolutionary branching point (100 generations). As a result of temporary correlations between marker trait and ecological character, assortative mating increases, which in turn magnifies these correlations (generations 400 to 1090). This positive feedback eventually leads to speciation (1150 generations). Gray scales indicate the frequency of phenotypic combinations (black corresponds to the highest frequency). Source: Dieckmann and Doebeli (1999). (b) Combinations of standard deviations of carrying capacity and competition function that allow for adaptive speciation. Gray scales indicate the time to speciation (black corresponds to 0 generations and white to 20000 or more generations). In the corresponding asexual model, evolutionary branching happens for combinations that lie below the diagonal. Parameters are as in Figure 1 , with $l_{\mathrm{A} 1}=l_{\mathrm{A} 2}=5$. The asterisk in (b) shows the parameter combination used in (a).

observed in deterministic models. Notice that even though the linkage equilibrium in the second scenario is stable, it is surrounded by but a small basin of attraction. Once this small domain is left through fluctuations, the resultant linkage disequilibrium is deterministically and swiftly amplified by selection. This result highlights a trade-off involved in using deterministic population models (e.g., like the one in Drossel and McKane 2000), which cannot easily capture processes of symmetry breaking that crucially rely on finite fluctuations for their initiation. In the present context, deterministic models could, in principle, be used to assess whether an initially small but finite linkage disequilibrium becomes large enough to allow for speciation, but they cannot be used to investigate the initial appearance of linkage disequilibria through stochastic effects, and thus cannot capture the speciation process in full. Note that in the second scenario, parameter requirements for evolutionary branching in sexual populations are somewhat more restrictive than those in the asexual case; information on this is summarized in Figure 4b.

Dieckmann and Doebeli (1999) also showed that adaptive speciation in the second scenario occurs more rapidly when fewer loci underlie the three quantitative characters (Figures $5 \mathrm{~b}$ and 5c). This is because fewer loci allow larger phenotypic effects of drift and are thus more likely to trigger the process of selection-enhanced symmetry breaking. An exception to this general tendency occurs for the extreme case $l_{\mathrm{E}}=1$, where only a single diallelic locus codes for the ecological character, which results in no more than three ecological phenotypes. Ironically, it was this special case that the influential analysis by Felsenstein (1981) relied on. However, Felsenstein did not even have the opportunity to realize that sympatric speciation was possible, even for $l_{\mathrm{E}}=1$, since he restricted his attention to purely deterministic models. Felsenstein's conclusions - putting "two-allele" mechanisms of sympatric speciation into disrepute for the next two decades - thus originated from an unfortunate confluence of restrictive modeling assumptions. Since the relative effects of drift that triggered the selection-enhanced symmetry breaking are most pronounced when populations are small, waiting times until adaptive speciation in the second scenario grow as population size increases. In the first scenario, the effect of loci numbers on speciation speed exhibits a pattern roughly similar to that in the second scenario (without a special role for $l_{\mathrm{E}}=1$ ). Here, however, times to speciation are considerably shorter - on the order of hundreds, rather 
(a)

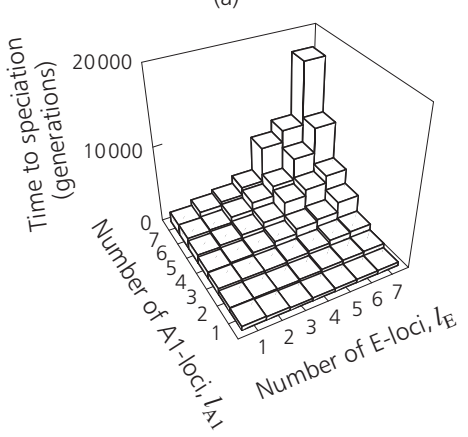

(b)



(c)

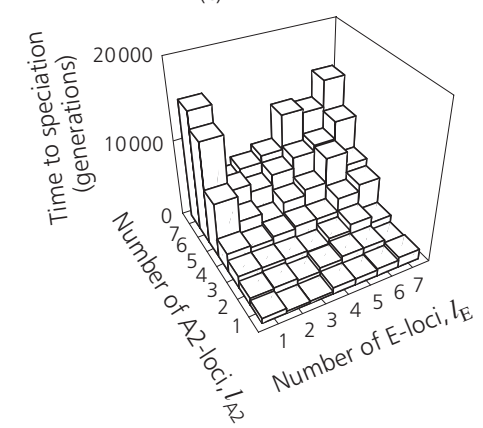

Figure 5 Dependence of time to speciation on the number of loci that affect the involved quantitative traits. (a) First scenario (assortative mating acts on ecological character); dependence on the number $l_{\mathrm{E}}$ of ecological loci and on the number $l_{\mathrm{A} 1}$ of mating loci. (b) Second scenario (assortative mating acts on marker trait); dependence on the number $l_{\mathrm{E}}$ of ecological loci and on the number $l_{\mathrm{A} 1}$ of mating loci for a fixed number of marker loci, $l_{\mathrm{A} 2}=5$. (c) Second scenario; dependence on the number $l_{\mathrm{E}}$ of ecological loci and on the number $l_{\mathrm{A} 2}$ of marker loci for a fixed number of mating loci, $l_{\mathrm{A} 1}=5$. Other parameters are as in Figure 1. Notice that the vertical scale in (a) differs from that in (b) and (c), and that - with the important exception of the special case $l_{\mathrm{E}}=1$ - more loci always imply slower speciation. Source $(b),(c)$ : Dieckmann and Doebeli (1999).

than thousands, of generations (Figure 5a). It also turns out that in the first scenario there is no significant effect of population size on speciation speed.

Notice that the model described above differs from the majority of previous theoretical studies on sympatric speciation in that it does not require two discrete patches or habitats, since resources in the continuum of types considered in the model are not spatially segregated. This means that there is no opportunity for populations to become allopatric (or parapatric) by very strong habitat choice or through very low migration. This makes it easier to appreciate that this model does not deal with allopatric (or parapatric) speciation in a "microalloptric" guise. Notice also that the results above remain qualitatively unchanged when assortative mating involves a cost of choosiness, as long as this cost is not too large. Such a cost of choosiness comes on top of the already present cost of rarity: when mating is assortative, rare phenotypes suffer from being chosen as mates with reduced probability. Obviously, large costs to assortative mating make its evolution less likely.

In summary, results for the two scenarios demonstrate that the genetic obstacles to sympatric speciation can, indeed, be overcome by sexual populations with stochastic multilocus genetics. What is more, the analysis above suggests sympatric speciation that requires either concordant or discordant allele substitutions can occur under realistic ecological and genetic conditions, and that even the degree of assortative mating gradually evolves as required for sympatric speciation. 


\section{Coevolutionary Adaptive Speciation in Sexual Populations}

While classic models used to study the processes of diversification focused on competition (e.g., MacArthur and Levins 1967), other types of ecological interactions have received less attention. We now illustrate how selection regimes that lead to evolutionary branching are expected to arise readily from a wide variety of different ecological interactions within and between species.

Specifically, Doebeli and Dieckmann (2000) demonstrated the potential for evolutionary branching in models for mutualism and for predator-prey interactions. In the models reviewed below, coevolutionary dynamics of quantitative characters in two separate species are driven by interspecific ecological interactions; frequency-dependent selection can then result in convergence to an evolutionary attractor at which either one or both species find themselves at fitness minima. This may lead to evolutionary branching in only one species, or in both species simultaneously, or in both species sequentially.

As shown by Law et al. (1997) and Kisdi (1999), combinations of intra- and interspecific competition can also lead to evolutionary branching. When competition is asymmetric, the resultant evolutionary dynamics are particularly prone to cycles of evolutionary branching and selectiondriven extinction.

\section{Adaptive speciation through mutualistic interactions}

Extending population dynamic studies by Vandermeer and Boucher (1978) and by Bever (1999), Doebeli and Dieckmann (2000) analyzed the potential for evolutionary branching in the following Lotka-Volterra system,

$$
\begin{aligned}
& \frac{\mathrm{d} n_{1 i}}{\mathrm{~d} t}=r_{1} n_{1 i}\left[1-\sum_{j} n_{1 j} / K_{1}\left(x_{1 i}\right)+\sum_{j} a_{1}\left(x_{1 i}, x_{2 j}\right) n_{2 j}\right], \\
& \frac{\mathrm{d} n_{2 i}}{\mathrm{~d} t}=r_{2} n_{2 i}\left[1-\sum_{j} n_{2 j} / K_{2}\left(x_{2 i}\right)+\sum_{j} a_{2}\left(x_{2 i}, x_{1 j}\right) n_{1 j}\right],
\end{aligned}
$$

where $n_{1 i}$ and $n_{2 i}$ are the population densities of mutualists in the two species with ecological characters $x_{1 i}$ and $x_{2 i}$, respectively; $r_{1}$ and $r_{2}$ are birth rates, and $K_{1}$ and $K_{2}$ are trait-dependent carrying capacities. The indices $i$ and $j$ range over all different ecological characters present in the two species, so that the second and third terms in the square brackets in Equations (2a) and (2b) determine, respectively, a death rate that results from intraspecific competition and an extra birth rate that results from interspecific mutualism. The mutualistic interaction between the two species is determined by the interaction functions $a_{1}$ and $a_{2}$, which depend on the ecological characters, as follows

$$
a_{1}\left(x_{1 i}, x_{2 j}\right)=a_{10} \frac{\alpha\left(x_{1 i}-x_{2 j}\right)}{\sum_{k} \alpha\left(x_{1 k}-x_{2 j}\right) n_{1 k}},
$$

with an analogous expression for $a_{2}\left(x_{2 i}, x_{1 j}\right)$. Here $\alpha$ is a normal distribution with mean 0 and variance $\sigma_{a}^{2}$-this shape implies that the mutualistic support between individuals decreases when their ecological characters move apart, with a tolerance given by $\sigma_{a}$. Following Kiester et al. (1984), it is assumed that the total mutualistic support an individual from one species provides to the other is fixed and given by $a_{10}$ and $a_{20}$, respectively. This assumption gives rise to the denominator in the equation above.

The trait-dependent carrying capacities are given by

$$
K_{1}\left(x_{1 i}\right)=K_{10}+K_{11} \beta\left(x_{1 i}\right),
$$

with an analogous expression for $K_{2}\left(x_{2 i}\right)$. Here $\beta$ is a normal distribution with maximum 1, mean $x_{01}$, and variance $\sigma_{K 1}^{2}$ : this shape implies that the carrying capacity in the first species possesses a 

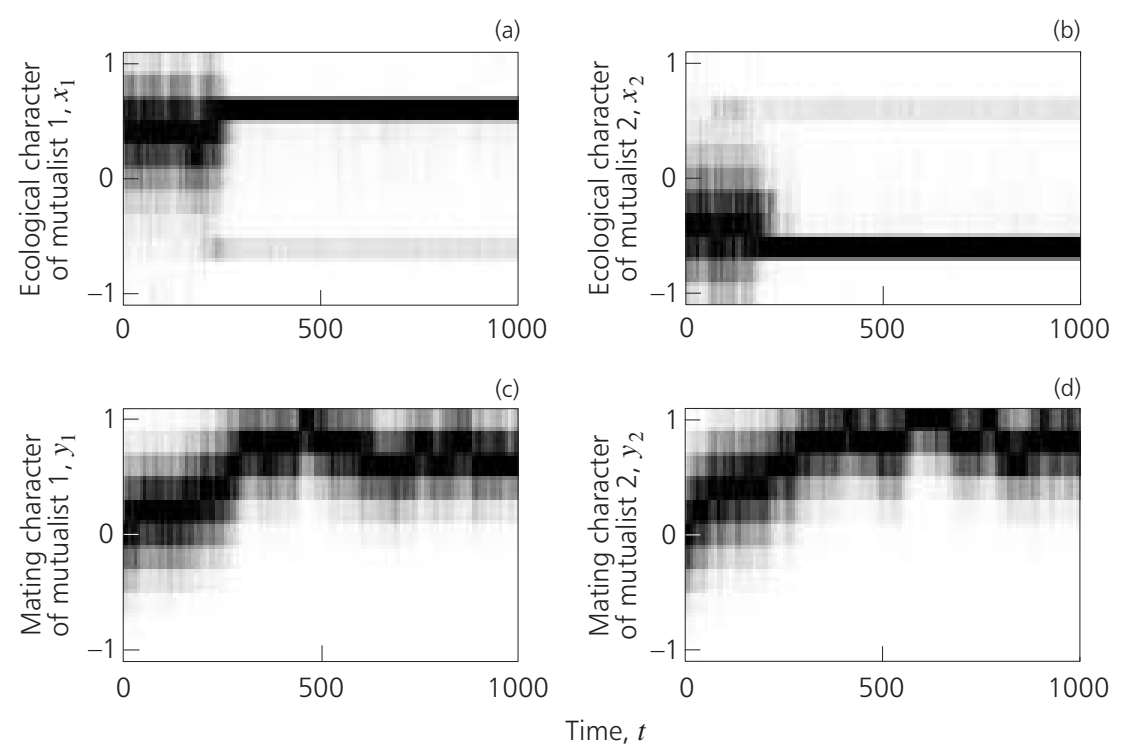

Figure 6 Adaptive speciation through mutualistic interactions. (a, c) Evolution of ecological characters jointly affects the compatibility of the two mutualists and their carrying capacities. (b, d) Evolution of the degree of assortative mating on the ecological characters. Both mutualists speciate simultaneously once their degree of assortative mating becomes high enough. Parameters: $r_{1}=r_{2}=1, K_{10}=K_{20}=300$, $K_{11}=K_{21}=400, x_{01}=-0.5, x_{02}=+0.5, \sigma_{K 1}=\sigma_{K 2}=0.5, a_{10}=a_{20}=0.00016, \sigma_{a}=0.2$, $l_{\mathrm{E}}=l_{\mathrm{A} 1}=5, u_{\mathrm{s}}=10^{-3}$. Source: Doebeli and Dieckmann $(2000)$.

baseline at the background level $K_{10}$, and increases, with a weighting factor $K_{11}$ and a tolerance $\sigma_{K 1}$, when the ecological character $x_{1 i}$ moves toward the carrying capacity's peak at $x_{01}$.

When the maxima of the mutualists' carrying capacities are different $\left(x_{01} \neq x_{02}\right)$, finite tolerances $\sigma_{a}, \sigma_{K 1}$, and $\sigma_{K 2}$ result in a tension between each species' advantage of adapting its ecological character to the peak of its own carrying capacity and the mutualistic benefit that it can reap from minimizing the distance between its ecological character and that of its mutualistic partner. When this tension is strong enough, gradual adaptations in $x_{1}$ and $x_{2}$ can trap either one or both species at fitness minima, and thus set the ecological stage for evolutionary branching. Introducing stochastic individual-based dynamics, multilocus genetics, and the evolution of assortative mating (as described in Section 2), Doebeli and Dieckmann (2000) confirmed that these situations can readily lead to sympatric speciation in sexual populations of the two mutualists.

As illustrated in Figure 6, the evolutionary outcome that results from such a speciation process is interesting: evolutionary branching creates two species pairs, in each of which one of the species is close to the peak of its carrying capacity and can thus provide much mutualistic support to the other species in the pair, which in turn is far away from the peak of its carrying capacity and therefore can give little support. Thus, in each of the two original species, speciation brings about a "supportive branch" and an "exploitative branch", and thereby breaks the community's symmetry prior to branching in each resultant species pair.

\section{Adaptive speciation through predator-prey interactions}

Evolutionary branching can also be induced by the ecological interactions between predators and their prey. Extending work by Brown and Vincent (1987, 1992), Marrow et al. (1992, 1996), Saloniemi (1993), Dieckmann et al. (1995), and Doebeli (1997), Doebeli and Dieckmann (2000) assumed that predation efficiency depends on two ecological characters, $x_{1}$ and $x_{2}$, the first in the prey and the other in the predator. These characters are scaled such that the interaction is the stronger the more similar these characters are. The ecological dynamics of a polymorphic 
(a)
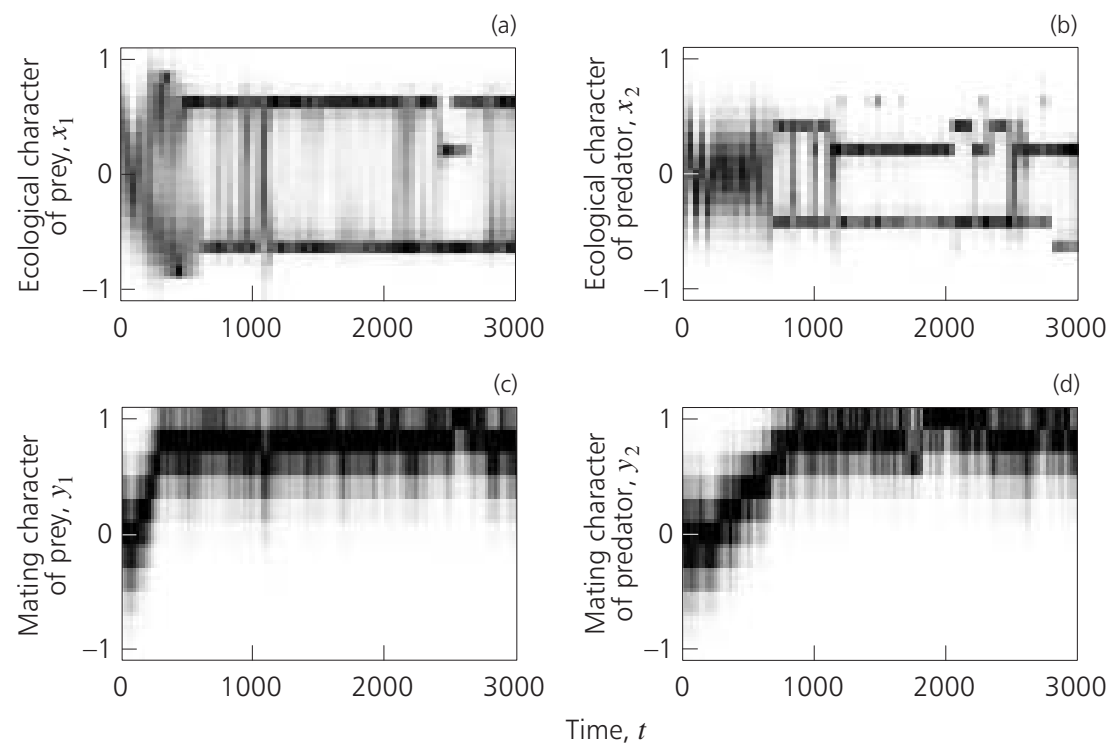

Figure 7 Adaptive speciation through predator-prey interactions. (a, c) Evolution of ecological characters jointly affects the prey harvesting efficiency of the predator and the carrying capacity of the prey. (b, d) Evolution of the degree of assortative mating on the ecological characters. After the prey has been evolutionarily chased and caught by the predator, and its degree of assortative mating has become high enough, it speciates, thus temporarily reducing the predator's harvesting efficiency. With the prey having split up, the predator's degree of assortative mating also increases, allowing it to speciate as well, which again increases its (now separate) harvesting efficiencies on each of the prey species. Parameters: $r=1$, $\delta=1, c=2, K_{0}=2000, x_{0}=0, \sigma_{K}=0.7, a_{0}=0.001, \sigma_{a}=0.4, l_{\mathrm{E}}=10, l_{\mathrm{A} 1}=5, u_{\mathrm{S}}=10^{-3}$. Source: Doebeli and Dieckmann (2000).

predator-prey community can be described by the Lotka-Volterra equations

$$
\begin{aligned}
\frac{\mathrm{d} n_{1 i}}{\mathrm{~d} t} & =r n_{1 i}\left[1-\sum_{j} n_{1 j} / K\left(x_{1 i}\right)-\sum_{j} a\left(x_{1 i}-x_{2 j}\right) n_{2 j}\right], \\
\frac{\mathrm{d} n_{2 i}}{\mathrm{~d} t} & =n_{2 i}\left[-\delta+c \sum_{j} a\left(x_{1 j}-x_{2 i}\right) n_{1 j}\right] .
\end{aligned}
$$

Here the predation efficiency $a$ depends on the character difference between prey and predator according to a normal distribution with maximum $a_{0}$, mean 0 , and variance $\sigma_{a}^{2}$, while the prey's carrying capacity function $K$ is a normal distribution with maximum $K_{0}$, mean $x_{0}$, and variance $\sigma_{K}^{2}$. The densities of prey and predator populations with ecological characters $x_{1 i}$ and $x_{2 i}$ are denoted by $n_{1 i}$ and $n_{2 i}$, respectively, while $r, \delta$, and $c$ measure the prey's intrinsic birth rate, the predator's intrinsic death rate, and the predator's conversion efficiency of prey resources. The indices $i$ and $j$ again range over all different ecological characters present in the two species. The second and third terms in the prey equation determine death rates that result, respectively, from intraspecific competition and from the predator's harvesting. Correspondingly, the sum in the predator equation determines a birth rate that results from harvesting the prey.

Notice that we do not assume any frequency dependence in the competitive interactions among the prey, which could be an independent cause for prey diversification even in the absence of predators. Yet, the existence of the predator imposes frequency-dependent selection on the prey through apparent competition, because common prey phenotypes have the disadvantage that the predator phenotype that preys upon them most efficiently is thriving. Doebeli and Dieckmann (2000) showed that this frequency dependence leads to evolutionary branching in the prey if predation efficiency decreases sufficiently fast with increasing distance between the ecological characters of prey and predator. 
Figure 7 shows the resultant process of sympatric speciation, based on the same extension to sexual populations as described in Section 2. Notice that in this example it is the primary evolutionary branching in the prey that induces evolutionary branching in the predator. This happens if the phenotypic distance between the two emerging prey species becomes large relative to the width $\sigma_{a}$ of the predation efficiency function (Doebeli and Dieckmann 2000). In this case the prey's evolution establishes the predator at a fitness minimum (see also Abrams and Matsuda 1996). The slightest polymorphism in the prey, maintained by mutation-selection balance, allows the coexistence of predator traits that straddle this fitness minimum, and thus allows the predator to undergo secondary evolutionary branching (É. Kisdi, personal communication). By contrast, if the two prey species remain sufficiently close to each other, the predator population does not experience disruptive selection and instead becomes a generalist (with predation efficiencies on each of the two emerging prey species significantly lower than the one it had on the single prey species prior to branching).

\section{Adaptive Speciation through Sexual Selection}

Darwin $(1859,1871)$ introduced the notion of sexual selection to explain the conspicuous sexual dimorphisms found in many species. Sexual selection arises from differences in reproductive success caused by competition for fertilization (Andersson 1994; see also Maynard Smith 1991); such selection can have both intrasexual and intersexual components. The former arise from the interaction between individuals of the same sex in the course of their competition for mates, while the latter originate from the interaction between the sexes when mates are actually chosen. Intersexual selection can be caused by sensory bias or drive (Ryan and Rand 1990; Endler 1992; Boughman 2001, 2002), which results in an organism's intrinsic preference for mates whose phenotypes stimulate its sensors where the sensors' sensitivity is highest.

Sexual selection is sometimes envisaged as being fundamentally different from natural selection. One reason might be Darwin's belief, still shared by some biologists, that only sexual selection can lead to the evolution of traits that appear irrelevant or even disadvantageous to their bearers, whereas "natural selection will never produce in a being anything injurious to itself" such that "any variation in the least degree injurious would be rigidly destroyed" (Darwin 1859). Today, we understand that both natural and sexual selection can harm a population by letting it converge to fitness minima (Sections 4.4 to 4.7 in Dieckmann et al. 2004 and Sections 2 and 3) and even by actively driving it to extinction (Matsuda and Abrams 1994a, 1994b; Ferrière 2000; Dercole et al. 2002; Gyllenberg et al. 2002; Dieckmann and Ferrière 2004). It is therefore appropriate to look upon the contrast between sexual and natural selection as being no more than a distinction that concerns the particular life-history component exposed to selection (Barnard 1998).

A key phenomenon that can arise from the interplay of intrasexual and intersexual selection is Fisher's "runaway process" (Fisher 1915, 1930). At the onset of such a process, some females for instance, because of a sensory bias - happen to mate preferentially with males that possess a certain trait or ornament: the resultant offspring inherits the genes both for the mother's preference and for the father's ornament, which results in a positive feedback that can fuel the further concerted evolution of male trait and female preference. While the male benefit that drives the runaway process is simply access to females, the female benefits can be more diverse - ranging from direct protection through the male to indirectly improved fitness for the female's offspring. Such indirect female benefits can arise either through an increased likelihood of mating with males that can afford to sport the preferred trait because of their above-average fitness ("handicap principle"; Zahavi 1975, 1977; Hamilton and Zuk 1982; Zahavi and Zahavi 1997) or through a greater attractiveness of their male offspring to other choosy females ("sexy son hypothesis"; Weatherhead and Robertson 1979). While the first effect relies on a correlation between male trait and male fitness, 
the second effect can involve a completely arbitrary, ecologically neutral male trait. A Fisherian runaway process could come to an end when genetic variance is exhausted (Kirkpatrick and Ryan 1991), developmental constraints are encountered, or costs start to exceed benefits - in particular when the male trait cannot be enhanced any further without exposing the male to strongly detrimental natural selection. With Fisher himself having provided a verbal argument only, mathematical models of Fisherian runaway were introduced and analyzed by O'Donald (1962, 1967, 1977, 1980), Lande (1980, 1981), and Kirkpatrick (1982b); see also Seger (1985b), Lande and Kirkpatrick (1988), Endler (1989), Pomiankowski et al. (1991), and Iwasa et al. (1991).

Mechanisms by which, not just one, but two or more Fisherian runaway processes can be triggered in a population are of particular interest in speciation theory, because of their potential to explain the evolution of prezygotic isolation. In the next two subsections we therefore briefly review speciation models based on the divergent evolution of mating preferences and preferred traits (see also Section 3.3 in Dieckmann et al. 2004). Speciation models based on sexual selection in spatially extended populations - including those by Lande (1982) and by Payne and Krakauer (1997) - are reviewed in Chapter 7 in Dieckmann et al. 2004. For a survey of empirical evidence that implicates sexual selection in speciation, see Panhuis et al. (2001).

\section{Adaptive speciation through mate competition}

Lande's original model of Fisherian runaway with a line of neutral equilibria (Lande 1981), as well as many subsequent investigations (e.g., Kirkpatrick 1982b and Seger 1985b), only led to genetic drift stochastically moving the coadapted combinations of male trait and female preference along this line. Soon it was discovered, however, that any slight cost to female choosiness reduced such neutral lines of equilibria to one or two asymptotically stable equilibrium points (Pomiankowski 1987; Bulmer 1989). With neutrality gone, populations that undergo separate Fisherian runaway can no longer diverge through genetic drift, which obliterates the potential of Lande's model to explain speciation. Also, being crucially based on neutral equilibria, Lande's speciation mechanism could provide no basis for the coexistence of the resultant species in stable polymorphisms. Other evolutionary forces were thus required to explain reproductive isolation between, and coexistence of, populations that undergo separate Fisherian runaway.

Such other forces were introduced in models by Turner and Burrows (1995) and by Higashi et al. (1999). Both studied the evolution through sexual selection of a polygenic male ornamental trait in finite populations of diploid individuals. The loci underlying the male trait were assumed to act additively and undergo free recombination, with intermediate male phenotypes living longer, and extreme phenotypes on either side of the male spectrum experiencing highest mortality. Populations were assumed to be polygynous, such that females examined many males before choosing one to mate with, and the resultant offspring had a 1:1 sex ratio.

In the study by Higashi et al. (1999), female preference for a particular male trait had the same genetic underpinning as the male trait itself, and females could examine the entire population of males before choosing a mate; mating probabilities decreased for males that did not meet their preference. Higashi et al. showed that, when starting from males with intermediate phenotypes and with females that preferred these males, both the male and the female character distribution could become bimodal, which resulted in reproductive isolation and thus speciation. The results by Higashi et al. (1999) are robust under the introduction of a cost of female choosiness, since, once the distributions of male trait and female preference have become bimodal, this cost can be compensated by the benefit of not producing unmated hybrid offspring. For a deterministic version of this model, see Takimoto et al. (2000).

By contrast, Turner and Burrows (1995) modeled female preference for either of two extreme male phenotypes through a single dominant allele; they allowed females to choose the bestmatching male out of a subset of males ("best-of- $n$ " rule), and assumed that Fisherian runaway 
had already brought about an extreme male phenotype together with the matching female preference. Turner and Burrows (1995) showed that, occasionally, a mutant female, which preferred the other end of the male spectrum to the resident females, could invade; this resulted, once again, in two reproductively isolated species with matching male traits and female preferences. The mutant females could invade since they were driven to mate with less extreme males, and therefore their male offspring enjoyed better survival than that of the resident females.

Both of these models are not without problems. The model by Turner and Burrows (1995) crucially relies on female preference being reversible through a single mutation - an assumption that, although perhaps justified in some natural systems, does not seem very general. The model by Higashi et al. (1999) suffers from the constraint that the initial distributions of male trait and female preference must be fine-tuned for speciation to occur. Unless female preference is more or less symmetrically distributed about the mode of the male distribution, no disruptive selection on males occurs and no speciation ensues. An interesting difference between the two approaches is that a reduced survival cost for extreme male phenotypes hinders speciation in the model by Turner and Burrows, but promotes speciation in the model by Higashi et al. (1999). Both models only work well if the females can examine a great number of males before committing themselves to a mate.

Fundamental problems of the sort just described would be overcome if plausible models of sexual selection could be devised that give rise to a (two-dimensional) evolutionary branching point (Box 4.3 in Dieckmann et al. 2004) for male trait and female preference. Evolution of the two sexual characters would then first converge to this point, at which both characters would experience frequency-dependent disruptive selection:

- Through its convergence stability an evolutionary branching point would solve the problem of the initial fine-tuning required in the model by Higashi et al. (1999).

- Through its lack of local evolutionary stability an evolutionary branching point would solve the problem of a large step in female preference being required in the model by Turner and Burrows (1995).

To have the option of encountering evolutionary branching points in models of sexual selection does not seem too far-fetched once it is realized that competition for mating partners is much akin to competition for ecological resources (Box 4.1 in Dieckmann et al. 2004 and Section 2). Specifically, there is a close formal resemblance between the distributions of an ecological resource and of female preference types available to males, and between a resource utilization spectrum and a function that describes the mating tolerance of females around a preferred male trait. Denoting, as in Section 2, the width of the former pair of distributions by $\sigma_{K}$ and of the latter pair by $\sigma_{a}$, recall that evolutionary branching of the ecological character happens if resource utilization is specific enough, $\sigma_{K}>\sigma_{a}$. Van Doorn and Weissing (2001) derived that, likewise, evolutionary branching of the male trait happens if female choice is specific enough, $\sigma_{K}>\sigma_{a} \sqrt{1+\sigma_{a}^{2} / \sigma_{\mathrm{s}}^{2}}$, where $1 / \sigma_{\mathrm{s}}$ measures the strength of stabilizing selection on the male trait at the evolutionary branching point. In the absence of such extra selection, this condition reduces to $\sigma_{K}>\sigma_{a}$, which confirms the strong analogy between evolutionary branching through mate competition and resource competition. Evolutionary branching through mate competition can also occur when male trait and female preference are high-dimensional genetic traits (Van Doorn et al. 2001).

The mechanism considered so far enables evolutionary branching in the one sex (usually the males) that experiences competition for mates. Yet, for speciation to occur, the other sex (usually the females) also has to undergo evolutionary branching. This means that frequency-dependent disruptive selection must act on female preference, which can occur in two ways (Van Doorn et al., unpublished): either through pleiotropic effects of frequency-dependent disruptive selection on an ecological character that evolves jointly (as in Van Doorn and Weissing 2001), or through female 
competition for males. In the former case speciation is not driven by sexual selection primarily; it also has to be underpinned by a suitable ecology. In the latter case, models ought to depart from the "typical sex-role assumption" that underlies most classic approaches to sexual selection. Under this assumption, only males are limited in their mating opportunities, whereas females have access to mates ad libitum, which implies that mate competition cannot cause frequency-dependent selection in females. Compared to reality, this remains an approximation, since, if there are far too many females around, their mating opportunities diminish. Especially if males have to engage in any time- or energy-intensive activities, like courtship or parental care, this approximation will often not be suitable.

Leaving the "typical sex-role assumption" aside, it can be shown that evolutionary branching of female preference becomes possible (Van Doorn et al., unpublished). This can lead, in principle, to the convergence of male trait and female preference, through directional selection, to a combination at which selection turns disruptive for both characters. This produces two Fisherian runaway processes that diverge from each other through frequency-dependent directional selection, which implies speciation. Frequency dependence means that the resultant species coexist in a stable polymorphism - a feature not encountered in many other models of speciation through sexual selection.

It turns out, however, that conditions for such two-dimensional evolutionary branching are quite restrictive (Van Doorn et al., unpublished). This is because parameter requirements for maletrait branching and female-preference branching do not overlap unless some additional source of frequency-dependent selection, other than mere competition for mates, is involved. This mutual exclusiveness arises since male fitness increases when a male mates with more females, whereas in the same situation female fitness decreases, which translates into opposing selective forces: when it pays males to diversify and undergo evolutionary branching, females experience stabilizing selection, and vice versa (Van Doorn et al., unpublished).

These results underscore the utility of investigating speciation by sexual selection through analysis of the potential for evolutionary branching, and highlight how the two sexes can first converge to and then escape from fitness minima induced by sexual selection. Adaptive speciation driven by sexual selection clearly is possible - even though perhaps less likely than previously believed.

\section{Adaptive speciation through sexual conflict}

Sexual conflict arises when traits that enhance the reproductive success of one sex reduce reproductive success of the other sex. It is appreciated increasingly that sexual conflict can be a strong driving force of evolutionary dynamics (Parker and Partridge 1998; Gavrilets 2000a; Martin and Hosken 2003). In particular, sexual conflict can lead to an evolutionary chase between the sexes, an idea substantiated both empirically (Arnquist and Rowe 2002) and theoretically (Gavrilets 2000a). Here we review a recent model by Gavrilets and Waxman (2002), which shows that sexual conflict over mating rates can give rise to adaptive speciation. This happens if, rather than staging a unidirectional evolutionary escape from males, females diversify into separate genotypic clusters. This can generate disruptive selection in the males, and so cause adaptive speciation with each female cluster being chased by a separate male cluster. The two pairs of genotypic clusters then represent incipient species.

Gavrilets and Waxman (2002) consider a sexual haploid population in which a multi-allelic locus A1 with alleles $x_{1}=0, \pm 1, \pm 2, \ldots$ determines female mating characteristics, while a multiallelic locus A2 with alleles $x_{2}=0, \pm 1, \pm 2, \ldots$ determines male mating characteristics. The probability that a female with allele $x_{1}$ is compatible with a male that has allele $x_{2}$ is given by a normal function $a\left(x_{1}-x_{2}\right)=\exp \left(-\frac{1}{2}\left(x_{1}-x_{2}\right)^{2} / \sigma_{a}^{2}\right)$, which peaks at 0 and has variance $\sigma_{a}^{2}$. The weighted number of males compatible with a female that has allele $x_{1 i}$ is then $\tilde{n}_{2 i}=\sum_{j} a\left(x_{2 j}-\right.$ $\left.x_{1 i}\right) n_{2 j}$, where $n_{2 j}$ is the number of males with allele $x_{2 j}$ and the sum extends over all male alleles 
present in the population. It is assumed that the reproductive success of a female with allele $x_{1 i}$ is a function $W_{1}\left(\tilde{n}_{2 i}\right)$ with a maximum at some intermediate value $\breve{n}_{2}$ - which means that too few as well as too many matings are detrimental to female reproductive success. Too many matings can be harmful if males employ aggressive mating strategies that damage the female, and it is this assumption that leads to sexual conflict in the model. Notice that this model is based on the "typical sex-role assumption" of limiting females and limited males. It also assumes that a reduced compatibility between males and females is sufficient to prevent the females from the harmful consequences of excessive mating (Panhuis et al. 2001).

To determine male reproductive success is more involved, because the success of a male depends not only on the frequency with which the various female alleles $x_{1 i}$ occur in the population, but also on the number of males $\tilde{n}_{2 i}$ compatible with these female alleles, as well as on the females' reproductive success $W_{1}\left(\tilde{n}_{2 i}\right)$. Details can be found in Gavrilets and Waxman (2002), which also presents the equations that govern the dynamics of allele frequencies in males and females. For the present purpose it suffices to indicate the important conceptual components of this model:

- Reproductive success in both males and females depends on the genotypic composition of the population (i.e., reproductive success is frequency dependent).

- In a population in which females are monomorphic for allele $x_{1}$, it is best for males to carry allele $x_{2}=x_{1}$.

- In a population in which males are monomorphic for allele $x_{2}$, it is best for females to carry allele $x_{1}=x_{2} \pm \Delta x$, where the optimal distance $\Delta x$ is determined by the functions $a$ and $W_{1}$.

This situation leads to an evolutionary chase of females by males. Male alleles that increase male compatibility with females are favored, while female alleles that move the number $\tilde{n}_{2}$ of compatible males closer to the optimal value $\check{n}_{2}$ are favored. This means that, evolutionarily, females tend to escape from males, which in turn try to catch up with them. Under certain conditions (for details, see Gavrilets 2000a), this can result in a continual evolutionary chase, as illustrated in Figure 8a, which schematically depicts the evolutionary dynamics of the average allelic values $x_{1}$ and $x_{2}$ in females and males. Depending on the initial conditions, these values evolutionarily converge on one of two lines, $x_{2}=x_{1} \pm \delta x$, and the evolutionary chase moves them along these lines toward $\pm \infty$; these two cases are highlighted as Chase ${ }_{+}$and Chase $e_{-}$in Figure 8a. The allelic distance between males and females thus equilibrates at a positive value $\delta x$ that is larger than the optimal distance 0 for males, but smaller than the optimal distance $\Delta x$ for females: $0<\delta x<\Delta x$.

Gavrilets and Waxman (2002) show that when selection on females is reduced by increasing $\check{n}_{2}$, and when mutation rates are increased, the evolutionary chase can be interrupted by diversification in the females through an evolutionary "tunneling" effect: a population of females that escapes from the males in one mutational direction at an average distance $+\delta x$ can occasionally give rise to successful mutant female alleles that are at a distance larger than $-\delta x$ from the current males, and thus escape from the males in the other direction. As only the absolute value, and not the direction of the distance to the males, is important, these mutant female alleles have similar reproductive success as the resident female allele. Such evolutionary tunneling can lead to the establishment of a polymorphism in the females.

In general, evolutionary tunneling happens whenever a fitness valley is crossed by a single, large mutational step. Figure 8b illustrates this through a pairwise invasibility plot for the female alleles, portrayed at any point in time throughout the evolutionary chase. In the situation shown, the population consists of males that are monomorphic for allelic value $x_{2}$. For any resident females allele $x_{1}$, all mutant female alleles $x_{1}^{\prime}$ that increase the distance to the males to a value closer to $\Delta x$ can invade (depicted in gray). For resident females alleles $x_{1}$ close to $x_{2}$, this means that two groups of mutant female alleles $x_{1}^{\prime}$ can invade: 
(a)

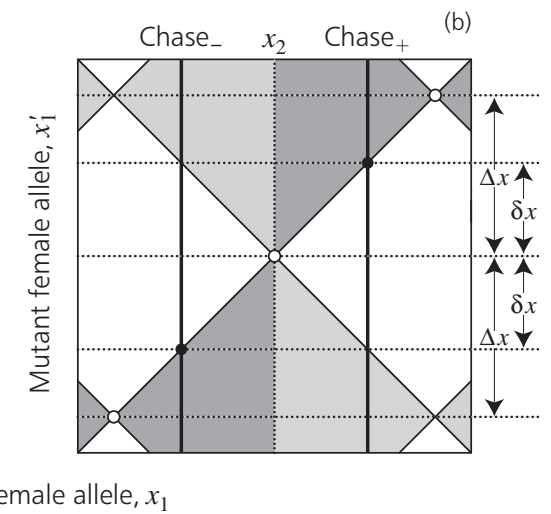

Figure 8 Adaptive speciation through sexual conflict. (a) Evolutionary chase of females by males. Any initial combination of female alleles $x_{1}$ and male alleles $x_{2}$ quickly evolves toward $x_{2}=x_{1} \pm \delta x$, which results in an intermediate level of male-female compatibility. The evolved distance $\delta x$ lies between the optimal distance 0 for males and the optimal distance $\Delta x$ for females, $0<\delta x<\Delta x$, and leads to the two alternative configurations of evolutionary chase shown by the two thick lines. Source: Gavrilets (2000a). (b) Pairwise invasibility plot for female alleles $\left(x_{1}^{\prime}, x_{1}\right)$ when the resident male allele is $x_{2}$. Combinations $\left(x_{1}^{\prime}, x_{1}\right)$ of mutant and resident female alleles for which the mutant can invade are highlighted in gray (light gray indicates those mutants that can be reached only through evolutionary tunneling). The two alternative relative positions of resident female alleles expected after some period of evolutionary chase are marked by thick lines. The two evolutionary singularities (indicated by the intersection points of the boundaries of the gray areas) in the lower-left and upper-right corners of the diagram represent locally evolutionarily stable female strategies $x_{1}=x_{2} \pm \Delta x$ for the given male trait $x_{2}$, while the evolutionary singularity in the diagram's center represents the combination $x_{1}=x_{2}$, which would be optimal for the males and acts as a repellor for evolution in the female trait.

- A first range of mutant female alleles $x_{1}^{\prime}$ that is immediately adjacent to $x_{1}$ (dark gray in Figure 8b) can invade.

- A second range of mutant female alleles $x_{1}^{\prime}$ that is situated on the opposite side of $x_{2}$ relative to the resident female allele $x_{1}$ (light gray in Figure 8b) can invade.

For example, if the distance of the resident female allele to the males is $\delta x$, as is expected after the evolutionary chase has settled on the line $x_{2}=x_{1}+\delta x$, female mutant alleles that are either a bit larger than $x_{1}$ or a bit smaller than $x_{2}-\delta x$ can invade (the latter case requires evolutionary tunneling). Likewise, for $x_{2}=x_{1}-\delta x$, female mutant alleles can invade that are either a bit smaller than $x_{1}$ or a bit larger than $x_{2}+\delta x$ (the latter again through tunneling). The preceding two cases are highlighted, respectively, as Chase ${ }_{+}$and Chase $e_{-}$in Figure 8b.

For the evolving population with allelic values $x_{2}$ in males and $x_{2}+\delta x$ in females it is clear that - as long as selection on females is sufficiently weak (so that $\delta x$ is small) and mutation rates and step sizes are large enough (so that mutations of sufficient size are available) - it is likely that female mutant alleles will appear in the population that allow for evolutionary tunneling. Once this happens, the female population becomes polymorphic, and consists of two female allelic clusters on either side of the single male cluster (Gavrilets and Waxman 2002). Scope for the stable coexistence of two female alleles (which is not evident from the monomorphic analysis in Figure $8 \mathrm{~b}$ ) is created by any slight degree of polymorphism in the male population that leads to frequency dependence in the females: if one of the female branches becomes more abundant, this is counteracted by the growth of the corresponding tail in the distribution of males. After diversification in the females, selection ceases to be directional in the males, and the evolutionary chase comes to a halt. Trapped between two equidistant female clusters the males can experience a situation that Gavrilets and Waxman (2002) liken to that of Buridan's Ass, forever trapped in the middle of two equally appealing bales of hay. 
However, if the distance between the female clusters and the trapped males is large enough, the male situation is not evolutionarily stable. More precisely, if $d$ is the allelic distance between each of the two female clusters and the males, then the males experience disruptive selection for mating compatibility if $\mathrm{d}^{2} a / \mathrm{d} d^{2}>0$ (Gavrilets and Waxman 2002). In this case, the males also diversify evolutionarily and form two clusters, each of which starts a new evolutionary chase with the corresponding female cluster. Once these separate chases have progressed far enough, the males that chase one female cluster become incompatible with the females from the other cluster, and vice versa: sympatric speciation has occurred. The whole process of trapping males through evolutionary tunneling in females and subsequent adaptive male diversification that leads to speciation can repeat itself within the newly emerging species (Gavrilets and Waxman 2002).

In sum, evolution in the above model is characterized by three phases:

- First is a phase of evolutionary chase between males and females, which takes the females close to the evolutionarily unstable singularity in the center of Figure $8 b$.

- Second, evolutionary tunneling in the females leads to a state in which males are trapped in the middle of two female clusters, thus ending the evolutionary chase. This tunneling effect results from mutational chance events and does not occur in the limit of very small and very rare mutations.

- Third, males diversify if the allelic distance between the two female clusters is large enough to generate a fitness minimum for the trapped males.

The model by Gavrilets and Waxman (2002) thus shows how sexual conflict can explain the dynamic emergence of disruptive selection through frequency-dependent interactions between the two sexes, and hence how sexual conflict can give rise to adaptive speciation. Notice the evolutionary dynamics of sequential evolutionary branching, first in females and then in males, that occurs in this model is similar to that exhibited by the coevolutionary predator-prey model discussed in Section 3.

Interestingly, in the high-dimensional trait spaces sexual selection is likely to operate in, evolutionary tunneling across fitness valleys might not even be required: the larger the number of dimensions, the higher the likelihood that these valleys do not actually have to be trespassed by large mutational jumps, but can instead be circumvented by sequences of small mutational steps [following an "extra-dimensional bypass" (Conrad 1990) along a "neutral network" (Schuster 1996), as explained in detail in Section 6.6 in Dieckmann et al. 2004 on "holey adaptive landscapes" (Gavrilets 1997)].

More generally, however, we think that more work is needed to determine whether sequential evolutionary branching is, indeed, a likely mechanism for adaptive speciation through sexual selection. There are two reasons for this:

- As for any other speciation mechanism, segregation and recombination are bound to destroy the bimodality required in the two sexes (Section 2). The corresponding problems are, of course, avoided when there is no segregation or recombination, as in the model of Gavrilets and Waxman (2002), which uses haploid genetics (no segregation) and a single locus for the traits involved (no recombination).

- When evolutionary branching in the two sexes is sequential, rather than simultaneous, the establishment of a sufficient degree of reproductive isolation between the incipient species is particularly difficult: if only females exhibit a bimodal distribution of their mating trait, while males are still unimodal, too much gene flow between the two female modes is likely to occur via the male population because of segregation and recombination, which thus collapses the female bimodality (G.S. van Doorn, personal communication). 
It is thus an interesting open question as to how robust the results by Gavrilets and Waxman (2002) are when more realistic genetic assumptions are allowed. Consequently, mechanisms of sympatric speciation through sexual selection based on mate competition, as described in the previous subsection - involving frequency-dependent selection in both sexes and based on simultaneous evolutionary branching - may turn out to be of wider relevance.

\section{Concluding Comments}

Key challenges in the theory of sympatric speciation are twofold. First, ecological conditions must induce disruptive selection in such a way that the evolving population does not become monomorphic for one of the favored phenotypes. Second, given such ecological conditions, the mating system must evolve such that reproductive isolation ensues between the phenotypes favored by disruptive selection. In the history of the theory of sympatric speciation, positions have shifted as to the rank of these two difficulties. In this chapter, we present an integrative framework with which to study sympatric speciation that simultaneously addresses both issues.

The ecological dynamics considered in Sections 2 and 3 - diverse as they are as to featured detail - share one common characteristic: they all give rise to evolutionary branching points as described by the theory of adaptive dynamics. Such ecological settings naturally allow for directional selection to converge to fitness minima (Rosenzweig 1978; Eshel and Motro 1981; Eshel 1983; Taylor 1989; Christiansen 1991; Brown and Pavlovic 1992; Metz et al. 1992, 1996; Abrams et al. 1993a; Geritz et al. 1997, 1998) where disruptive selection can initiate sympatric speciation. As highlighted by Tregenza and Butlin (1999), "This provides an explanation for the nagging problem in other models of how the initial population comes to be in a state in which all phenotypes are intermediate and adaptation to the environment is suboptimal." Or, as put by Kawecki (Section 3.2 in Dieckmann et al. 2004), "sympatric speciation requires that the intermediate genotypes are first superior and then inferior to the original genotype. This apparent contradiction was seen as a major argument against sympatric speciation (Mayr 1963; Futuyma and Mayer 1980). A solution to this conundrum lies in the frequency dependence of fitness, which causes the adaptive landscape to change dynamically as the population evolves." There are four important points to appreciate here:

- A great variety of generic ecological settings robustly induces evolutionary branching points.

- Evolutionary branching points explain how populations come to be situated at fitness minima, without the need for exceedingly unlikely external events.

- Evolutionary branching points also retain populations at such minima for whatever time it takes them to evolutionarily overcome genetic constraints that prevent escape from such a trap.

- Since the underlying disruptive selection is not imposed externally, but rather is generated internally through frequency-dependent selection, no fine-tuning of conditions and parameters is required to stabilize the resulting dimorphism.

The genetic dynamics considered throughout this chapter illustrate that previous misgivings about the potential for sexual populations to escape disruptive selection by becoming bimodal in the selected trait may have been overrated. Both for "one-allele" and "two-allele" mechanisms of assortative mating, genetic constraints imposed by segregation and recombination are readily overcome in stochastic multilocus models, provided disruptive selection is not too weak. Felsenstein (1981) has conceded already that "It is not clear a priori whether the results found here are sensitive to the number of loci assumed involved in the traits." As described in Section 2, we now know that Felsenstein's hesitation was indeed justified: multilocus models of sympatric 
speciation do behave quite differently from earlier models that involved only one locus for each character. And when the traditional deterministic models are replaced by more realistic individualbased stochastic models, the scope for sympatric speciation widens even further. What is more, the stochastic multilocus models described in this chapter demonstrate that even when starting out from a population that is determined genetically to mate randomly, the degree of assortativeness can increase gradually because of the selection pressures encountered at an evolutionary branching point. This means that sympatric speciation in sexual populations can evolve completely from scratch, without the artificial assumption either that the population miraculously starts out situated at an externally imposed fitness minimum or that, from the very start, assortative mating occurs whenever ecologically different phenotypes encounter each other.

The reason why assortative mating evolves under branching conditions is easy to understand. Under frequency-dependent disruptive selection, segregation and/or recombination trap the population at a fitness minimum for as long as mating remains random. Assortative mating reduces the generation of intermediate offspring phenotypes from extreme parent phenotypes. As their offspring are thus less likely to exhibit a phenotype at the fitness minimum, individuals that mate assortatively are favored by natural selection. Once assortative mating has evolved, evolutionary branching can occur in sexual populations. In "one-allele" models of assortative mating, this mechanism alone allows for sympatric speciation. In "two-allele" models, which require the emergence of a linkage disequilibrium, the evolution of increasing levels of assortativeness additionally involves a process of selection-enhanced symmetry breaking (as illustrated in Figure 4).

A wide range of particular mechanisms for assortative mating exists in nature, and a large set of ecologically neutral marker phenotypes is always available for assortativeness to act on. It is thus important to realize that any single one of these mechanisms can enable a sexual population to escape from its trap at an evolutionary branching point. Viscous populations - in which a local component of a large population may succeed in escaping the fitness minimum through speciation, and subsequently conquer the population's remainder - further diminish the propensity of populations to remain stuck at evolutionary branching points. In some settings (including, most prominently, the evolution of host races), assortative mating even comes for free with the resource utilization of organisms. In addition, earlier rounds of sympatric speciation that involved evolutionary branching are bound to have left behind a legacy of assortative mating options, upon which subsequent speciation processes can freely draw; thus the escape of trapped populations is even more likely.

With the genetic constraints on sympatric speciation thus looking considerably less severe than many studies and discussions over the past few decades suggested, it is the ecological constraints that take center stage in determining the likelihood of sympatric speciation. Evaluation of these ecological constraints reduces to no more than addressing the simple and general question as to whether or not a particular ecology features an evolutionary branching point. As this question has been answered affirmatively for a wide variety of fundamental ecological models, not much doubt can remain that, from the vantage point of contemporary theory, sympatric speciation is entirely feasible. Since the speciation mechanism described here enables a population to escape from a fitness minimum, the speciation process itself must be considered adaptive. In the words of Seger (1985a), "sympatric speciation could provide, figuratively speaking, an easy way out of a difficult ecological and developmental bind." In general, adaptive speciation unfolds as a solution to a particular evolutionary problem posed by a population's frequency-dependent ecology (Chapters 1 and 19 in Dieckmann et al. 2004). This means that adaptive speciation is just as good a paradigm for asexual species as it is for sexual species - no fundamentally disparate mechanism have to be invoked to explain why both asexual and sexual species come in discrete chunks rather than in an unstructured continuum (Dobzhansky 1951). 
It is exciting to realize that models of speciation by sexual selection also exhibit the characteristics of adaptive speciation. As shown in Section 4, an evolutionarily unstable singularity (Box 4.4 in Dieckmann et al. 2004) is always implicated in the reviewed models. We must thus regard processes of adaptive speciation driven by natural and sexual selection as two sides of the same coin: whether the traits that experience frequency-dependent disruptive selection affect a population's ecological interactions or its mating system is a matter of detail, not of principle. The eco-evolutionary feedback that can cause evolutionary branching (Chapter 1 in Dieckmann et al. 2004) may operate equally well through components of an individual's environment affecting the abundance of preferred resources or mates. Yet, adaptive speciation through sexual selection involves some additional problems, different from those relevant to adaptive speciation through natural selection. The reason is that under pure mate competition it pays males to diversify whenever females experience stabilizing selection, and vice versa (since, when a male mates with more females, male fitness increases, but female fitness decreases; Van Doorn et al., unpublished). As explained in Section 4, this means that simultaneous evolutionary branching in both sexes cannot happen unless, as well as mate competition, additional sources of frequency-dependent selection contribute to the process. A notable alternative is adaptive speciation through sexual conflict: here evolutionary branching in the two sexes happens sequentially rather than simultaneously, driven by mate competition under the divergent mating interests of the two sexes.

We close this chapter by highlighting some compelling directions for future research. On the theoretical side, we should try to do better justice to the complexity of evolving mating systems and study models that can simultaneously describe adaptations in at least four different quantitative traits: the ecological character under frequency-dependent disruptive selection, a male marker trait, a female trait that determines which male trait females prefer to mate with, and a fourth trait that specifies the choosiness or specificity of female preference. While the analyses by Kondrashov and Kondrashov (1999) and by Dieckmann and Doebeli (1999) have already covered two different subsets, each containing three out of these four traits, investigation of a full four-trait model is pending. Also, studies that target specific types of assortative mating in more mechanistic detail are needed to enhance our understanding of how these affect the likelihood of adaptive speciation. Eventually, we need to bring together studies of the spatial patterns and temporal processes involved in speciation. Most speciation processes, strictly speaking, are parapatric to a larger or smaller extent - ranging from, as the extremes along a realistic continuum, cases with almost no gene flow between incipient species to cases with almost complete mixing. For this reason (and also since the traditional divide between allopatric and sympatric speciation is based on spatial pattern) it is compelling to incorporate a spatial dimension into the models described in this chapter - a program that is carried out in Chapter 7 in Dieckmann et al. 2004.

On the empirical side, unbiased assessments of the relative frequency at which (mostly) sympatric or allopatric speciation process occur in nature pose an essential challenge. A recent review by Turelli et al. (2001) concluded that "comparative analyses (Barraclough and Nee 2001) show that, in several taxa, the most recently evolved species generally have allopatric ranges, supporting Mayr's view that allopatric speciation might be most common." We believe that, unfortunately, such conclusions are as yet unwarranted: if sympatric mechanisms can induce spatial segregation between emerging species readily (as demonstrated in Section 7.4 in Dieckmann et al. 2004; Doebeli and Dieckmann 2003), abutting distributions of even recently evolved species can offer no clues as to the underlying speciation mechanism - unless a geographic barrier to gene flow at the time of speciation is demonstrated explicitly. It therefore seems fruitful to refocus the timehonored debate about the sympatric versus allopatric mode on the specific mechanisms that drive natural speciation processes, and, in particular, on evaluating the extent to which these processes are adaptive (Chapters 1 and 19 in Dieckmann et al. 2004). For such purposes, new approaches and methods will be needed (Berlocher 1998) to prevent problematic implicit assumptions - about 
how past processes can be inferred from present patterns - causing a bias in such assessments in the future. We suggest that this can be achieved reliably only by combining spatially explicit genetic, ecological, and environmental data and by focusing attention on speciation in gestation. Steps toward this ambitious goal are discussed in Chapter 15 in Dieckmann et al. 2004.

\section{References}

Abrams PA \& Matsuda H (1996). Fitness minimization and dynamic instability as a consequence of predator-prey coevolution. Evolutionary Ecology 10:167-186

Abrams PA, Matsuda H \& Harada Y (1993). Evolutionarily unstable fitness maxima and stable fitness minima of continuous traits. Evolutionary Ecology 7:465-487

Andersson M (1994). Sexual Selection. Princeton, NJ, USA: Princeton University Press

Arnquist G \& Rowe L (2002). Antagonistic coevolution between the sexes in a group of insects. Nature 415:787-789

Barnard CJ (1998). Sexual selection. In The Encyclopedia of Ecology and Environmental Management, ed. Calow P, pp. 681-682. Oxford, UK: Blackwell Science

Barraclough TG \& Nee S (2001). Phylogenetics and speciation. Trends in Ecology and Evolution 16:391-399

Barraclough TG \& Vogler AP (2000). Detecting the geographical pattern of speciation from species-level phylogenies. The American Naturalist 155:419-434

Berlocher SH (1998). Can sympatric speciation via host or habitat shift be proven from phylogenetic and biogeographic evidence? In Endless Forms: Species and Speciation, eds Howard DJ \& Berlocher SH, pp. 99-113. New York, NY, USA: Oxford University Press

Berlocher SH \& Feder JL (2002). Sympatric speciation in phytophagous insects: Moving beyond controversy? Annual Review of Entomology 47:773-815

Bever JD (1999). Dynamics within mutualism and the maintenance of diversity: inference from a model of interguild frequency dependence. Ecology Letters 2:52-61

Boughman JW (2001). Divergent sexual selection enhances reproductive isolation in sticklebacks. Nature 411:944-948

Boughman JW (2002). How sensory drive can promote speciation. Trends in Ecology and Evolution 17:571-577

Bridle JR \& Jiggins CD (2000). Adaptive dynamics: Is speciation too easy? Trends in Ecology and Evolution 15:225-226

Brown JS \& Pavlovic NB (1992). Evolution in heterogeneous environments: Effects of migration on habitat specialization. Evolutionary Ecology 6:360-382.

Brown JS \& Vincent TL (1987). Predator-prey coevolution as an evolutionary game. In Applications of Control Theory in Ecology, ed. Cohen Y, pp. 83-101. Lecture Notes in Biomathematics 73. Berlin, Germany: Springer-Verlag

Brown JS \& Vincent TL (1992). Organization of predator-prey communities as an evolutionary game. Evolution 46:1269-1283

Bulmer M (1989). Structural instability of models of sexual selection. Theoretical Population Biology 35:195-206

Bush GL (1975). Modes of animal speciation. Annual Review of Ecology and Systematics 6:339364

Bush GL (1998). The conceptual radicalization of an evolutionary biologist. In Endless Forms: Species and Speciation, eds. Howard DJ \& Berlocher SH, pp. 425-438. New York, NY, USA: Oxford University Press

Christiansen FB (1991). On conditions for evolutionary stability for a continuously varying character. The American Naturalist 138:37-50 
Christiansen FB \& Feldman MW (1975). Subdivided populations: A review of the one- and twolocus deterministic theory. Theoretical Population Biology 7:13-38

Conrad M (1990). The geometry of evolution. Biosystems 24:61-81

Darwin C (1859). On the Origin of Species by Means of Natural Selection, or the Preservation of Favoured Races in the Struggle for Life. London, UK: John Murray

Darwin C (1871). The Descent of Man, and Selection in Relation to Sex. London, UK: John Murray

Dercole F, Ferrière R \& Rinaldi S (2002). Ecological bistability and evolutionary reversals under asymmetrical competition. Evolution 56:1081-1090

Dickinson H \& Antonovics J (1973). Theoretical considerations of sympatric divergence. The American Naturalist 107:256-274

Dieckmann U \& Doebeli M (1999). On the origin of species by sympatric speciation. Nature 400:354-357

Dieckmann U, Doebeli M, Metz JAJ \& Tautz D (2004). Adaptive Speciation. Cambridge, UK: Cambridge University Press.

Dieckmann U \& Ferrière R (2004). Adaptive dynamics and evolving biodiversity. In Evolutionary Conservation Biology, eds. Ferrière R, Dieckmann U \& Couvet D, pp. 188-230. Cambridge, UK: Cambridge University Press

Dieckmann U, Marrow P \& Law R (1995). Evolutionary cycling in predator-prey interactions: Population dynamics and the Red Queen. Journal of Theoretical Biology 176:91-102

Dobzhansky T (1951). Genetics and the Origin of Species. Third Edition. New York, NY, USA: Columbia University Press

Doebeli M (1996). A quantitative genetic competition model for sympatric speciation. Journal of Evolutionary Biology 9:893-909

Doebeli M (1997). Genetic variation and the persistence of predator-prey interactions in the Nicholson-Bailey model. Journal of Theoretical Biology 188:109-120

Doebeli M \& Dieckmann U (2000). Evolutionary branching and sympatric speciation caused by different types of ecological interactions. The American Naturalist 156:S77-S101

Drossel B \& McKane A (2000). Competitive speciation in quantitative genetic models. Journal of Theoretical Biology 204:467-478

Endler JA (1989). Conceptual and other problems in speciation. In Speciation and its Consequences, eds. Otte D \& Endler JA, pp. 625-648. Sunderland, MA, USA: Sinauer Associates Inc.

Endler JA (1992). Signals, signal conditions, and the direction of evolution. The American Naturalist 139:S125-S153

Eshel I (1983). Evolutionary and continuous stability. Journal of Theoretical Biology 103:99-111

Eshel I \& Motro U (1981). Kin selection and strong stability of mutual help. Theoretical Population Biology 19:420-433

Feder JL, Chilcote CA \& Bush GL (1988). Genetic differentiation between sympatric host races of the apple maggot fly Rhagoletis Pomonella. Nature 336:61-64

Felsenstein J (1981). Skepticism towards Santa Rosalia, or why are there so few kinds of animals? Evolution 35:124-138

Ferrière R (2000). Adaptive responses to environmental threats: evolutionary suicide, insurance, and rescue. Options Spring 2000, pp. 12-16. Laxenburg, Austria: International Institute for Applied Systems Analysis

Fisher RA (1915). The evolution of sexual preference. Eugenics Review 7:184-192

Fisher RA (1930). The Genetical Theory of Natural Selection. Oxford, UK: Clarendon Press

Futuyma DJ \& Mayer GC (1980). Non-allopatric speciation in animals. Systematic Zoology 29:254-271 
Gavrilets S (1997). Evolution and speciation on holey adaptive landscapes. Trends in Ecology and Evolution 12:307-312

Gavrilets S (2000). Rapid evolution of reproductive barriers driven by sexual conflict. Nature 403:886-889

Gavrilets S \& Waxman D (2002). Sympatric speciation by sexual conflict. Proceedings of the National Academy of Sciences of the USA 99:10533-10538

Geritz SAH \& Kisdi É (2000). Adaptive dynamics in diploid, sexual populations and the evolution of reproductive isolation. Proceedings of the Royal Society of London B 267:1671-1678

Geritz SAH, Metz JAJ, Kisdi É \& Meszéna G (1997). Dynamics of adaptation and evolutionary branching. Physical Review Letters 78:2024-2027

Geritz SAH, Kisdi É, Meszéna G \& Metz JAJ (1998). Evolutionarily singular strategies and the adaptive growth and branching of the evolutionary tree. Evolutionary Ecology 12:35-57

Gyllenberg M, Parvinen K \& Dieckmann U (2002). Evolutionary suicide and evolution of dispersal in structured metapopulations. Journal of Mathematical Biology 45:79-105

Hamilton WD \& Zuk M (1982). Heritable true fitness and bright birds: A role for parasites? Science 218:384-387

Higashi M, Takimoto G \& Yamamura N (1999). Sympatric speciation by sexual selection. Nature 402:523-526

Iwasa Y, Pomiankowski A \& Nee S (1991). The evolution of costly mate preferences. II. The "handicap" principle. Evolution 45:1431-1442

Johnson PA, Hoppensteadt FC, Smith JJ \& Bush GL (1996). Conditions for sympatric speciation: A diploid model incorporating habitat fidelity and non-habitat assortative mating. Evolutionary Ecology 10:187-205

Kawecki TJ (1996). Sympatric speciation driven by beneficial mutations. Proceedings of the Royal Society of London B 263:1515-1520

Kawecki TJ (1997). Sympatric speciation via habitat specialization driven by deleterious mutations. Evolution 51:1749-1761

Kiester AR, Lande R \& Schemske DW (1984). Models of coevolution and speciation in plants and their pollinators. The American Naturalist 124:220-243

Kirkpatrick M (1982). Sexual selection and the evolution of female choice. Evolution 36:1-12

Kirkpatrick M \& Ryan MJ (1991). The paradox of the lek and the evolution of mating preferences. Nature 350:33-38

Kisdi É (1999). Evolutionary branching under asymmetric competition. Journal of Theoretical Biology 197:149-162

Kisdi É (2001). Long-term adaptive diversity in Levene-type models. Evolutionary Ecology Research 3:721-727

Kisdi É \& SAH Geritz (1999). Adaptive dynamics in allele space: evolution of genetic polymorphism by small mutations in a heterogeneous environment. Evolution 53:993-1008

Kondrashov AS (1983a). Multilocus model of sympatric speciation I. One character. Theoretical Population Biology 24:121-135

Kondrashov AS (1983b). Multilocus model of sympatric speciation II. Two characters. Theoretical Population Biology 24:136-144

Kondrashov AS (1986). Multilocus model of sympatric speciation. III. Computer simulations. Theoretical Population Biology 29:1-15

Kondrashov AS \& Kondrashov FA (1999). Interactions among quantitative traits in the course of sympatric speciation. Nature 400:351-354

Kondrashov AS \& Mina MV (1986). Sympatric speciation: when is it possible? Biological Journal of the Linnean Society 27:201-223 
Kondrashov AS \& Shpak M (1998). On the origin of species by means of assortative mating. Proceedings of the Royal Society of London B 265:2273-2278

Lande R (1980). Sexual dimorphism, sexual selection, and adaptation in polygenic characters. Evolution 34:292-305

Lande R (1981). Models of speciation by sexual selection on polygenic traits. Proceedings of the National Academy of Sciences of the USA 78:3721-3725

Lande R (1982). Rapid origin of sexual isolation and character divergence in a cline. Evolution 36:213-223

Lande R \& Kirkpatrick M (1988). Ecological speciation by sexual selection. Journal of Theoretical Biology 133:85-98

Law R, Marrow P \& Dieckmann U (1997). On evolution under asymmetric competition. Evolutionary Ecology 11:485-501

Levene H (1953). Genetic equilibrium when more than one ecological niche is available. The American Naturalist 87:331-333

MacArthur R \& Levins R (1967). The limiting similarity, convergence, and divergence of coexisting species. The American Naturalist 101:377-385

Marrow P, Law R \& Cannings C (1992). The coevolution of predator-prey interactions: ESSs and Red Queen dynamics. Proceedings of the Royal Society of London B 250:133-141

Marrow P, Dieckmann U \& Law R (1996). Evolutionary dynamics of predator-prey systems: An ecological perspective. Journal of Mathematical Biology 34:556-578

Martin OY \& Hosken DJ (2003). The evolution of reproductive isolation through sexual conflict. Nature 423:979-982

Matessi C, Gimelfarb A \& Gavrilets S (2001). Long term buildup of reproductive isolation promoted by disruptive selection: How far does it go? Selection 2:41-64

Matsuda H \& Abrams PA (1994a). Runaway evolution to self-extinction under asymmetrical competition. Evolution 48:1764-1772

Matsuda H \& Abrams PA (1994b). Timid consumers - self-extinction due to adaptive change in foraging and anti-predator effort. Theoretical Population Biology 45:76-91

Maynard Smith J (1966). Sympatric speciation. The American Naturalist 100:637-650

Maynard Smith J (1991). Theories of sexual selection. Trends in Ecology and Evolution 6:146151

Mayr E (1963). Animal Species and Evolution. Cambridge, MA, USA: Harvard University Press

Metz JAJ, Nisbet RM \& Geritz SAH (1992). How should we define "fitness" for general ecological scenarios? Trends in Ecology and Evolution 7:198-202

Metz JAJ, Geritz SAH, Meszéna G, Jacobs FJA \& Van Heerwaarden JS (1996). Adaptive dynamics, a geometrical study of the consequences of nearly faithful reproduction. In Stochastic and Spatial Structures of Dynamical Systems, eds. Van Strien SJ \& Verduyn Lunel SM, pp. 183-231. Amsterdam, Netherlands: North-Holland

O'Donald P (1962). The theory of sexual selection. Heredity 22:499-518

O'Donald P (1967). A general model of sexual and natural selection. Theoretical Population Biology 12:298-334

O’Donald P (1977). Theoretical aspects of sexual selection. Theoretical Population Biology 12:298-334

O’Donald P (1980). Genetic Models of Sexual Selection. Cambridge, UK: Cambridge University Press

Panhuis T, Butlin R, Zuk M \& Tregenza T (2001). Sexual selection and speciation. Trends in Ecology and Evolution 16:364-371

Parker GA \& Partridge L (1998). Sexual conflict and speciation. Philosophical Transactions of the Royal Society of London B 353:261-274 
Pomiankowski A (1987). The costs of choice in sexual selection. Journal of Theoretical Biology 128:195-218

Pomiankowski A, Iwasa Y \& Nee S (1991). The evolution of costly mate preferences. I. Fisher and biased mutation. Evolution 45:1422-1430

Rosenzweig ML (1978). Competitive speciation. Biological Journal of the Linnean Society 10:275-289

Ryan MJ \& Rand AS (1990). The sensory basis of sexual selection for complex calls in the túngara frog, Physalaemus pustulosus (sexual selection for sensory exploitation). Evolution 44:305-314

Saloniemi I (1993). A coevolutionary predator-prey model with quantitative characters. The American Naturalist 141:880-896

Schliewen UK, Rassmann K, Markmann M, Markert J, Kocher TD \& Tautz D (2001). Genetic and ecological divergence of a monophyletic species pair under fully sympatric conditions in Lake Ejagham, Cameroon. Molecular Ecology 10:1471-1488

Schuster P (1996). How does complexity arise in evolution? Complexity 2:22-30

Seger J (1985a). Intraspecific resource competition as a cause of sympatric speciation. In Evolution: Essays in Honour of John Maynard Smith, eds. Greenwood PJ, Harvey PH \& Slatkin M, pp. 43-53. Cambridge, UK: Cambridge University Press

Seger J (1985b). Unifying genetic models for the evolution of female choice. Evolution 39:11851193

Takimoto G, Higashi M \& Yamamura N (2000). A deterministic genetic model for sympatric speciation by sexual selection. Evolution 54:1870-1881

Taylor PD (1989). Evolutionary stability in one-parameter models under weak selection. Theoretical Population Biology 36:125-143

Tregenza T \& Butlin RK (1999). Speciation without isolation. Nature 400:311-312

Turelli M, Barton NH \& Coyne JA (2001). Theory and speciation. Trends in Ecology and Evolution 16:330-343

Turner GF \& Burrows MT (1995). Sympatric speciation through sexual selection. Proceedings of the Royal Society of London B 260:287-292

Udovic D (1980). Frequency-dependent selection, disruptive selection, and the evolution of reproductive isolation. The American Naturalist 116:621-641

Van Doorn GS \& Weissing FJ (2001). Ecological versus sexual selection models of sympatric speciation. Selection 2:17-40

Van Doorn GS, Luttikhuizen PC \& Weissing FJ (2001). Sexual selection at the protein level drives the extraordinary divergence of sex-related genes during sympatric speciation. Proceedings of the Royal Society of London B 268:2155-2161

Van Doorn GS, Dieckmann U \& Weissing FJ. Sympatric speciation by sexual selection: A critical re-evaluation. Unpublished.

Vandermeer JH \& Boucher DH (1978). Varieties of mutualistic interactions in population models. Journal of Theoretical Biology 74:549-558

Weatherhead PL \& Robertson RJ (1979). Offspring quality and the polygyny threshold: "The sexy son hypothesis". The American Naturalist 113:201-208

Zahavi A (1975). Mate selection - a selection for a handicap. Journal of Theoretical Biology 53:205-214

Zahavi A (1977). The cost of honesty. Further remarks on the handicap principle. Journal of Theoretical Biology 67:603-605

Zahavi A \& Zahavi A (1997). The Handicap Principle. Oxford, UK: Oxford University Press 